\title{
A Contribution to a Theory of Mechanochemical Pathways by means of Newton Trajectories
}

\author{
Wolfgang Quapp ${ }^{\star, 1}$. Josep Maria Bofill ${ }^{\#, 2}$
}

Received: February 10, 2016 / Revised: Mart 22 / Accepted:

Keywords Effective potential energy surface, Mechanochemistry, Newton trajectory, Barrier breakdown, Saddle, Intermediate, Reaction Path

Subject area: general interest in mechano-chemistry

\begin{abstract}
The reaction path is a central subject in theoretical chemistry. It is a pathway imagined on the potential energy surface (PES). It provides a one-dimensional description of a chemical reaction in an $N$-dimensional configuration space. Additionally, one can apply mechanical stress in a defined direction to the molecule and generate an effective PES. Changes for minima and saddle points by the stress are described by Newton trajectories on the original PES. The barrier of a reaction fully breaks down for the maximal value of the norm of the gradient of the PES along a pulling Newton trajectory. This point is named barrier breakdown point (BBP). We discuss topologically different, 2-dimensional examples for this model to understand and classify the mechanochemistry of molecules.
\end{abstract}

\section{Introduction}

The potential energy surface (PES) and the reaction path (RP) are basic concepts of many theoretical chemistry models. The RP is a one-dimensional description of a chemical reaction through a sequence of molecular geometries in an $N$-dimensional configuration space. We use $N=3 n-6$ for the number of non-redundant internal coordinates, and $n$ is the number of the atoms of the molecular system. An often used RP model is the distinguished coordinate [1]. It was later generalized as the distinguished coordinate path (DCP) $[2,3,4]$ and was finally refined as Newton trajectory (NT) $[3,4,5,6,7,8,9,10]$. For this type of RP holds that at every point of the curve the gradient of the PES points into the same direction, a direction of a prescribed search vector. This property is the central idea of mechanochemical stress models of a molecule. It is the reason that NTs should be taken into account for mechanochemical problems.

The paper is organized as follows: first we repeat the known model of mechanochemical stress for a PES and explain the use of NTs in the model. Then we introduce a definition of the optimal pulling direction. With many 2-dimensional test surfaces we explain and classify the impact of stress on the topography of the modified PES. We begin with uncoupled potential bowls, and end with competing reaction channels and the emergence of intermediates under stress. (The examples are work under progress: we will keep adding new topics by self-study.) The paper finishes with a discussion and a conclusion.

\section{Newton Trajectories are Pathways of Mechanochemical Transformations}

In the last years, the phenomena of the action of a mechanical stress over a molecular system have motivated experimental and theoretical researches $[11,12,13,14,15,16,17,18,19,20]$. According to the IUPAC terminology, one

\footnotetext{
${ }^{1}$ Leipzig University, Mathematisches Institut, Universität Leipzig, PF 100920, D-04009 Leipzig, Germany

${ }^{\star}$ E-mail: quapp@uni-leipzig.de

${ }^{2}$ Universitat de Barcelona, Departament de Química Orgànica, Universitat de Barcelona, and Institut de Química Teòrica i Computacional, Universitat de Barcelona, (IQTCUB), Martí i Franquès, 1, 08028 Barcelona Spain

\#E-mail: jmbofill@ub.net
} 
defines a mechanochemical reaction as "a chemical reaction that is induced by mechanical energy" [17]. Here, we will quantify molecular stress geometrically. We will discuss the problem how an external force couples to various reaction mechanisms [21]; where basically, the generally accepted model [20,22] consists in a first order perturbation on the associated PES of the unperturbed molecular system due to a stress or pulling force, $\mathbf{f}$

$$
V_{f}(\mathbf{r})=V(\mathbf{r})-\mathbf{f}^{T} \mathbf{R}
$$

where the symbol $T$ means transposition of a vector or a matrix. Of course, the linear perturbation is the simplest model [23] at all; perhaps it is an oversimplification, compare Ref.[15]. However, in the following we will use the linear model, and for this simple model we obtain some deep insights into mechanochemical scenarios. $\mathbf{R}$ in Eq. 1 is the change of a distance between the two pulling points of the molecule [24]. It will be associated with one of the internal coordinates [24] or a linear combination of them. We assume here that the plane of two intrinsic coordinates is the stage where the pulling works. Note that the theory and the use of NTs are not restricted to 2-dimensional examples. We can assume that $d R \approx d r$ for a coordinate change in direction of $\mathbf{R}$, and $d r$ is a linear combination of the coordinates of our 2-dimensional examples. The potential $V_{f}(\mathbf{r})$ can be seen as an effective PES. Since the external force, $\mathbf{f}$, stays constant it does not depend on the position. Due to this external force, the stationary points are located at different positions on the effective potential [25] with respect to the unperturbed potential, $V(\mathbf{r})$, where it holds $\nabla_{\mathbf{r}} V(\mathbf{r})=\mathbf{g}(\mathbf{r})=\mathbf{0}$. The stationary points on the effective potential have to satisfy the analogous condition, $\nabla_{\mathbf{r}} V_{f}(\mathbf{r})=\mathbf{0}$, depicting new and displaced stationary points. With the direction $d R \approx d r$ there follows for the new minimum with Eq.1

$$
\nabla_{\mathbf{r}} V_{f}(\mathbf{r})=\mathbf{0}=\mathbf{g}-\mathbf{f}
$$

thus one searches a point where the gradient of the original PES, $\mathbf{g}$, has to be equal to the mechanochemical force, f, being the force that induces the chemical process. An early proposal to use an equation like Eq.2 was given by Kliesch [26] which was an impulse to develop the application of NTs for PESs in chemistry [3,4]. If the mechanical stress in a defined direction is $\mathbf{f}=F \mathbf{l}$ with a fixed unit vector, $\mathbf{l}$, then it is $\mathbf{l}=\mathbf{g} /|\mathbf{g}|$ and $F=|\mathbf{g}|$ is the magnitude. We also use the alternate form for the solution of the Eq.2 by the projector equation $[3,4]$

$$
\left(\mathrm{U}-1 \mathbf{l}^{T}\right) \mathrm{g}=\mathbf{0}
$$

where $\mathbf{U}$ is the unit matrix. The equation has to hold unattached from the uncomfortable norm, $|\mathbf{g}|$, and it means nothing else that $\mathbf{g}$ and $\mathbf{l}$ are parallel.

Expanding Eq.2 in a Taylor sum with two summands only, we get [25] for the location of the new extremal value, $\mathbf{r}_{e x}$

$$
\mathbf{r}_{e x, f}=\mathbf{r}_{e x, 0}+\mathbf{H}^{-1}\left(\mathbf{r}_{e x, 0}\right) \mathbf{f}
$$

where $\mathbf{H}$ is the Hessian of the PES. For the shift of a minimum, the shift is positive, to the right hand side of increasing $\mathbf{r}$, where the saddle is shifted to the left, if the pulling direction goes along the saddle col. Then the shift is negative because the col eigenvalue of the Hessian is negative. Of course, the model clearly assumes that $\mathbf{r}_{e x, f}$ moves with the force, $\mathbf{f}$. That is an improvement to an older assumption [27]. However, the reduction of the barrier hight is the dominating contribution [28].

Figure 1 is an example for a 1-dimensional pulling scenario [29,30]. Along the x-axis we represent a Morse potential curve, $V(x)=4.5(1.0-\operatorname{Exp}[-x+1.0])^{2}$ where $x=1$ is the equilibrium point, and $D_{e}=4.5$ is the dissociation energy in any units. Additionally, there are two effective curves $\mathrm{V}_{f 1}(x)=V(x)-x$ and $\mathrm{V}_{f 2}(x)=V(x)-2.275 x$, compare Ref.[31]. The $\mathrm{V}_{f 2}$ has a shoulder at the $|\mathbf{g}|_{\max }$ point, at $x=1.693$ of the original curve, $\mathbf{V}$. If the force, $F=|\mathbf{f}|$, is so high that the SP disappeared into a shoulder, then the mechanochemical task is fulfilled: the pulling force enforced the chemical reaction, the reaction barrier broke down [32]. It is the barrier breakdown point (BBP). The reader might also think about a bond breaking point (BBP). - Note: this holds for the very simple model ansatz of Eq.(1). And of course, by thermal fluctuations, a real molecule will leave the minimum well before the BBP point is catched at a shoulder of the effective PES [33].

Now we treat a fixed direction of $\mathbf{l}$, but different forces, $F$, in the higher dimensional case [20,27]. Thus, for pointto-point changing forces, $F$, we should get the 'reaction path following force displaced stationary points (FDSPs)' [22]. They are on the solution of the differential equation of Branin [34] which we can use in $N=3 n-6$ nonredundant coordinates [35]

$$
\frac{d \mathbf{r}}{d t}= \pm \mathbf{A}(\mathbf{r}) \mathbf{g}(\mathbf{r})
$$




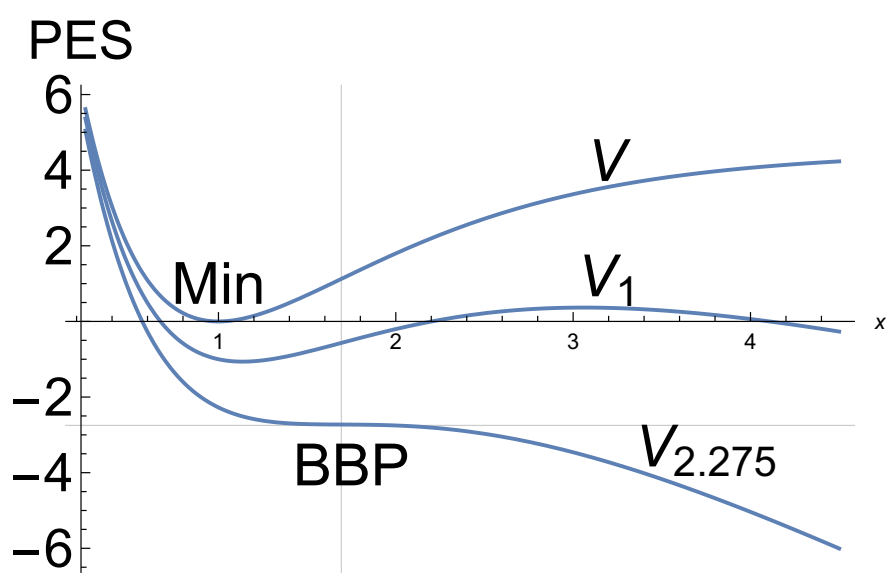

Fig. 1 1D Morse potential, V, over the $x$-axis: the upper curve. Below are two other effective potential curves with an increasing force, f. The minimum moves along increasing $x$-values, where the SP moves along decreasing values. The lowest potential is the BBP case: minimum and SP coalesce to a shoulder. The former barrier is broken.

$t$ is a curve parameter and the matrix $\mathbf{A}$ is constructed as a product of the determinant of the Hessian with the inverse Hessian,

$$
\mathbf{A}=\operatorname{Det}(\mathbf{H}) \mathbf{H}^{-1}
$$

The determinant is a number. It does not change the direction of the vector $\mathbf{H}^{-1} \mathbf{g}$ used on the right hand side of Eq.4. However, it removes the singularity of $\mathbf{H}^{-1}$ emerging on the way to the SP. Of course, the way from the minimum to the SP is always anharmonic including the singular inverse Hessian at $|\mathbf{g}|_{\text {max }}$. The matrix $\mathbf{A}$ is named the desingularized inverse Hessian, or the adjoint to $\mathbf{H}$. Since one uses curvilinear internal coordinates, one can additionally apply the metric form [36] of Eq.5.

The signs ' \pm ' are used to allow the curve to go uphill from a minimum, or downhill from an SP. Curves $\mathbf{r}(t)$ satisfying this expression are called Newton trajectories (NT). A property of Eq.5 is that the gradient at every curve point always points into the same direction, $\mathbf{l}$, if it had this direction at an initial point. Then holds the parallelity $\mathbf{l}|| \mathbf{g}$ throughout the path $[3,4]$. To see this fact briefly, we consider the behavior of the gradient $\mathbf{g}(\mathbf{r}(t))$ along a solution, $\mathbf{r}(t)$. We obtain [4] for a change of $\mathbf{g}$ with $t$

$$
\frac{d \mathbf{g}}{d t}=\mathbf{H}(\mathbf{r}) \frac{d \mathbf{r}}{d t}= \pm \mathbf{H} \mathbf{A} \mathbf{g}= \pm \operatorname{Det}(\mathbf{H}) \mathbf{g}
$$

where Eq.5 is used. Thus, the gradient $\mathbf{g}$ changes proportionally to $\mathbf{g}$ itself. This means that the direction of $\mathbf{g}$ does not change. It is invariant along the solution and fulfills Eq. 2 for corresponding values of $|\mathbf{f}|=F$. It also means that the solution is a DCP, as well as a solution of the projector equation Eq.3.

The solution curve of the Branin equation is a regular curve from a point near the minimum to an SP if no valley-ridge inflection point is crossed [4]. The Branin equation [34] (5) is a well-know model for RPs [3,4]. This RP model is especially used here for the FDSPs: for every special force, $\mathbf{f}$, we get a moved stationary point of the new effective potential, $V_{f}$, but this point is represented on the NT of the original PES, $V$, where this NT is used for the pulling direction. An NT can start near both kinds of stationary points, minimums or saddle points in every direction. And it goes (usually, if no valley-ridge inflection point is met) from a minimum to an SP of index one, or vice versa (the last is the 'index theorem' [37]).

We assume that the mechanical force $\mathbf{f}$ points into a constant direction, $\mathbf{l}$, that is $\mathbf{f}=F \mathbf{l}$. If $\mathbf{r}$ is a point of the FDSPs curve then we can set $\mathbf{r}=\mathbf{r}(t)$ being $t$ the parameter that characterizes this curve for variable forces, $F$. It is like the Branin equation. Now, we differentiate the projector Eq.3 with respect to $t$, and we obtain $[4,7]$

$$
\left(\mathbf{U}-1 \mathbf{l}^{T}\right) \mathbf{H} \frac{d \mathbf{r}}{d t}=\mathbf{0}
$$

This is a second expression of the tangent of the FDSPs curve. It is the basic expression of the reduced gradient following curve (RGF), or NT, derived many years ago [4,7] for the projector equation. For higher dimensions of the $\mathrm{PES}$, this equation is better to track numerically than the Branin equation.

If one moves on the path of FDSPs one has to increase the norm of the force, $F$, beginning at the stationary points: there is a part of the pathway from the minimum uphill, and a part from the SP downhill, compare Fig.1. Anywhere, 
if the force increases further and further, the two parts meet. Here the norm of the gradient has its maximum. Thus, the curvature of the PES along the corresponding NT is zero. If we are on a straight valley path, say in $x$-direction, then one eigenvector of the Hessian points along the path, and at the meeting point of the lower and the upper part of the FDSPs we find out that the corresponding eigenvalue of the Hessian is zero. There the barrier of the original PES disappears. On the effective PES, $V_{f}$, with the maximal force, the SP disappears, and the pulling force realizes the reaction. The meeting point is a 'catastrophe point' [20,22,24]; this is a word of the well known theory of Thom $[38,39]$. We propose to name it more chemical: barrier breakdown point (BBP). Its necessary mathematical formula is $[40]$

$$
\operatorname{det}(\mathbf{H})=\mathbf{0}
$$

Note that $\mathbf{H}$ is always the Hessian of the original, zero-force PES, because the model Eqs.1 and 2 do not influence the calculation of the Hessian [42]. To prepare the idea of the proof of condition Eq.9 we remark that the BBP is a turning point of the function $|\mathbf{g}|$ along the NT.

\section{A general proof of Eq.9 for BBPs:}

We search the turning point (TP) of the function $|\mathbf{g}|$ along the NT: the directional derivative of $|\mathbf{g}|$ along the NT has to be zero.

$$
\frac{d}{d t} \sqrt{\mathbf{g}^{T} \mathbf{g}}=\frac{2}{\sqrt{\mathbf{g}^{T} \mathbf{g}}} \mathbf{g}^{T} \mathbf{H} \frac{d \mathbf{r}}{d t}= \pm \frac{2}{\sqrt{\mathbf{g}^{T} \mathbf{g}}} \mathbf{g}^{T} \mathbf{H A g}= \pm 2 \sqrt{\mathbf{g}^{T} \mathbf{g}} \operatorname{det}(\mathbf{H})=0
$$

where first the concept of directional derivative is used and second the tangent vector, $d \mathbf{r} / d t$, is replaced by the definition given in Eq.5. The proof is finished: it is easy because we have the tool of NTs at hand.

If the pulling direction, $\mathbf{l}$, points along the straight line of the reaction coordinate from the minimum to the SP then we have the 1-dimensional case of Fig.1 embedded in an $N$-dimensional PES. This case is, so to say, the 'simple' one $[32,33]$. However, note that the pulling problem is really high-dimensional $[20,27,30]$. In Section 4 , we will treat many 2-dimensional examples to discuss the question: what happens to an allowed NT, representing the FDSPs, to a direction outside the main valley?

\section{The Optimal Pulling Direction}

An important Corollary of the present theory is the following result.

\section{Corollary}

The mechanical force to be applied to the molecule of interest is $\mathbf{f}=F \mathbf{l}$ where $F=\sqrt{\mathbf{g}^{T} \mathbf{g}}$. All the regular NTs that leave the minimum and arrive the same SP cross at least once a $\operatorname{Det}(\mathbf{H})=0$-line. The first $\operatorname{Det}(\mathbf{H})=0$-line that each NT crosses gives the BBP of this NT. The BBP is the point where $F$ is a maximum for this NT. If we compare all NTs of such a set, then the NT which gives the lowest value of $F$ is named the optimal NT. It coincides with a gradient extremal (GE) exactly at the intersection point with the $\operatorname{Det}(\mathbf{H})=0$-line. By definition holds that at each point of a GE curve the gradient norm, $|\mathbf{g}|$, is stationary, very often it is minimal, within a variation of each isopotential hypersurface of the PES traversed by this GE curve [41]. As a consequence each point of the GE satisfies the expression $\mathbf{H} \mathbf{g}=\lambda \mathbf{g}$, thus the gradient is an eigenvector of the Hessian matrix. At the point where a GE curve crosses the $\operatorname{Det}(\mathbf{H})=0$-line we have $\lambda=0$ and due to this fact $\mathbf{H} \mathbf{g}=\mathbf{0}$, otherwise it is a VRI point. Following the same procedure as in Eq.10 and using the previous result we have that at the point of the $\operatorname{Det}(\mathbf{H})=0$-line that intersects with a point of the GE curve the variation of $|\mathbf{g}|$ trough a curve obeys

$$
\frac{d}{d t} \sqrt{\mathbf{g}^{T} \mathbf{g}}=\frac{2}{\sqrt{\mathbf{g}^{T} \mathbf{g}}} \mathbf{g}^{T} \mathbf{H} \frac{d \mathbf{q}}{d t}=\frac{2}{\sqrt{\mathbf{g}^{T} \mathbf{g}}} \mathbf{0}^{T} \frac{d \mathbf{q}}{d t}=0
$$

Because the set of NTs starting from a minimum covers the PES region of interest, there has to be a special NT that at this point has $d \mathbf{q} / d t$ as tangent and $|\mathbf{g}|$ takes a maximal value along this curve and a minimal value with respect to the others NTs that cross this $\operatorname{Det}(\mathbf{H})=0$-line in a neighborhood. Both, the direction 1 evaluated from the normalized gradient vector of this BBP and the magnitude $F=|\mathbf{g}|$ define the minimal covalent mechanochemistry force, $\mathbf{f}$, to be applied. 


\section{Examples}

We illustrate the concept of using NTs in more detail, with quite different types of a test PES, in the following 2-dimensional examples. The two coordinates used, $(x, y)$, may be the plane of the two most important dimensions of a chemical reaction. All other remaining coordinates are projected out of the treatment. It is further assumed that an important part of the pulling vector acts in this $(x, y)$-plane in any linear combination [21]. The examples may be understood as topological extracts of chemical examples for pulling scenarios $[22,40,43]$ where the high molecular dimension of the problem is also projected into two intrinsic dimensions. Of course, this is a simplified model.
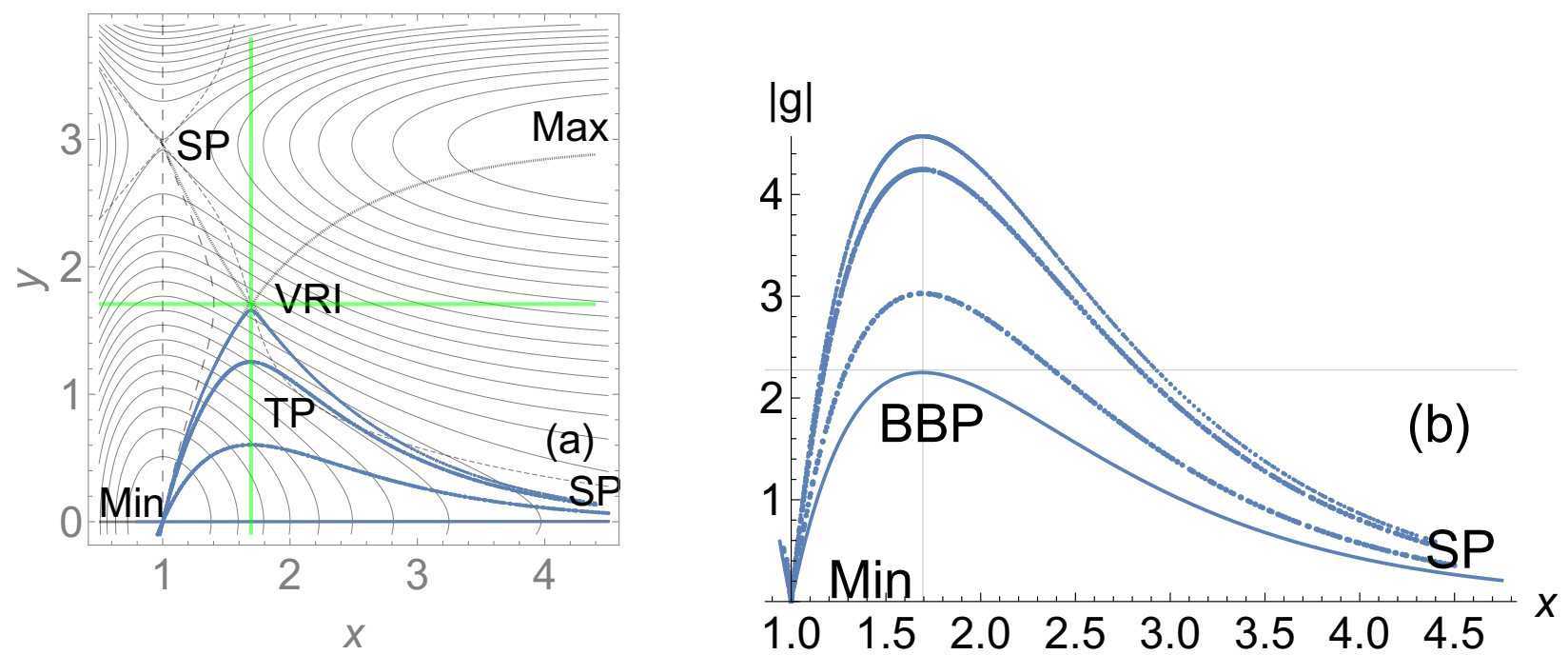

Fig. 2 (a) NTs on a 2D potential. In $x$-direction it is Morse, in $y$-direction it is a quartic potential. The blue, bold faced NTs go to the right hand SP of interest, the gray curve is a singular NT through the valley-ridge inflection (VRI) point, and the two dashed curves into the North direction go to the higher SP at $(1,3)$. Two blue NTs have two turning points (TP). The green cross of lines is the condition $\operatorname{Det}(\mathbf{H})=0$. It marks the BBPs on NTs. (b) Profiles of $|\mathbf{g}|$ over the four basic curves, the four blue NTs of panel (a). The curve over the $x$-axis, along the MEP, needs the smallest force to proceed to its end, the BBP. It represents the optimal pulling. The other curves need a higher force. All BBPs are here on a straight line, where $\operatorname{Det}(\mathbf{H})=0$ holds, at $x=1.695$. Order: the lower curve is the one over the MEP, the upper is the one over the singular NT through the VRI point.

Example 1

In Fig.2 we discuss the (trivial) straight valley case on a decoupled PES. The formula is given in Appendix (A1). A straight NT along the $x$-axis would be the 'simple' case: the pulling direction as the reaction coordinate [31]. However, we treat a full family of different NTs which connect the minimum with the Morse-SP on the $x$-axis. Four NTs of the family are drawn in blue in Fig.2(a). The zero of the pulling coordinate is assumed at $(0,0)$. We are interested in the norm of the gradient, $|\mathbf{g}|$, of the PES along the NTs. The relation $\operatorname{Det}(\mathbf{H})=0$ is fulfilled on straight lines on the used surface. It is the BBP condition and corresponds to the case of Ref.[40], Fig. 1(a) there. Always on the 'maximum' of the NT in the $(x, y)$ coordinates points the tangent of the NT in the $x$-direction. The norm of the gradient of the PES over the four blue basic curves is given in Fig.2(b). Note, the value of the PES at the VRI point, through which the 'highest', the singular NT leads, is higher than the value of the PES at the Morse-SP. One can follow the level lines, to see this. At the VRI point, the singular NT crosses the line $\operatorname{Det}(\mathbf{H})=0$ (green colored), as well as the convexity border of the PES (thin dashes) given by the Hirsch condition [44] $\mathbf{g}^{T} \mathbf{A} \mathbf{g}=0$. The proof of this formula is very simple. Analogous to the former proof of the BBP condition, Eq.10, we have to set to zero the directional derivation of $V$ along the NT. It is the given formula.

The VRI point is crossed by a singular NT with four branches. Two of its branches are the limit region for a full family of NTs which connect the minimum with the Morse-SP on the $x$-axis: they form the upper blue line with a kink at the VRI point. Other NTs with a still steeper slope, into the direction of the $y$-axis, belong to the other family of NTs which connect the minimum with the quartic SP on the $y$-axis. The VRI point discriminates the two families of NTs. 

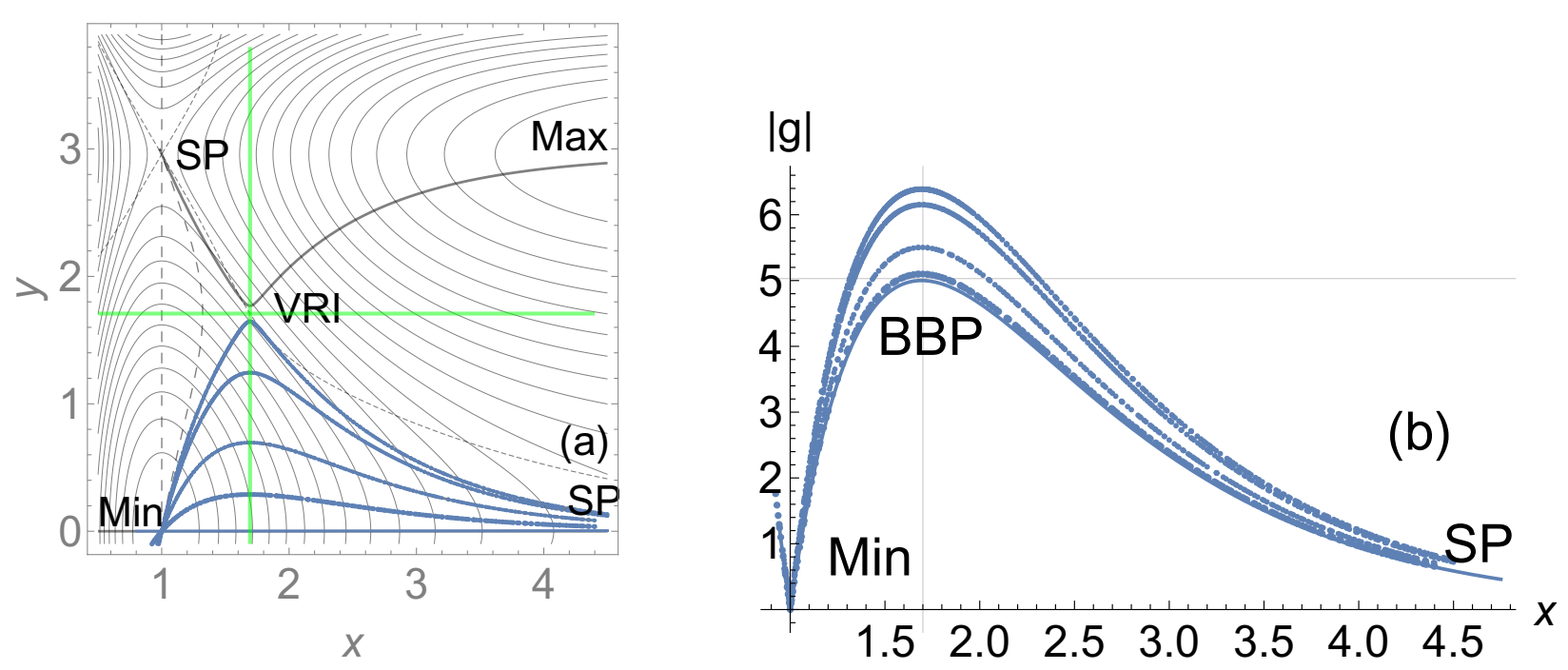

Fig. 3 (a) NTs on a 2D potential. In $x$-direction it is Morse, in $y$-direction it is a quartic potential, however, the Morse-SP is now higher. The blue NTs go to the right hand SP of interest, and the gray curve is a singular NT through the valley-ridge inflection (VRI) point. The two dashed curves into the North direction go to the lower SP in $y$-direction. The green cross of lines is the condition $\operatorname{Det}(\mathbf{H})=0$. (b) profiles of $|\mathbf{g}|$ over the blue basic NTs of the left panel. All BBPs are here on a straight line, where $\operatorname{Det}(\mathbf{H})=0$ holds, at $x=1.695$. Order: the lower curve is the one over MEP, the upper is the one over the singular NT through the VRI point.

There exist TPs on the outer NTs near the VRI. For the pulling model, the TPs do not influence the process of the overcoming of the barrier, see Fig.2(b).

Conclusion: NTs to other directions than the 'MEP' along the $x$-axis are possible but they need a higher force to enforce the reaction. Note that also the energy increases at the outer BBPs. Thus, the strength of the pulling force counts, but also the direction, to meet a BBP. Usually, there exists an optimal pulling direction.

\section{Example 2}

Now, we alternate the height of the two SPs of the decoupled example: we represent the PES by a next 2D toy surface, given in Appendix (A2). Here, the Morse-SP on the $x$-axis is the higher one. The qualitative picture stays at the same level; only some TPs of the NTs near the VRI disappear. We get the left Fig.3, and the profiles of $|\mathbf{g}|$ over the NTs to the Morse-SP become those of the right panel (b). If one compares Fig.3(b) and Fig.2(b), one has to conclude that here the BBPs are higher and they are closer together. Note that the lower SP on the $y$-axis does not disturb the pulling scenario along the $x$-axis.

\section{Example 3}

We again turn the relation of the two axes: the $x$-axis is a quartic/sixtic part of the PES, the $y$-axis is the Morse part; and the $x$-SP is lower than the Morse-SP. The minimum is flat in $x$-direction, but the PES becomes a steep slope near the right SP. We whant to enforce a reaction over the right SP by a pulling scenario. We get the Fig.4(a), and the profiles of function $|\mathbf{g}|$ over the NTs to the quartic/sixtic SP become that of Fig.4(b). The value of the PES at the VRI point, through which the 'highest', the singular NT leads, is higher than the value of the PES at the $x$-SP, but lower in comparison to the Morse-SP on the $y$-axis. One can follow the level lines, to see this. The BBPs on the different NTs to the $x$-SP (the blue parts of the NTs) are now nearer to the SP, and far from the minimum, see Fig.4(b). The BBPs are 'late' near the SP, in contrast to the former examples.

\section{Example 4}

What happens to a curvilinear valley from the minimum to the SP? We discuss this for a deep potential bowl with a double minimum, and a low lying SP in between [8,20,22], see the Appendix (A4) for the formula. To represent the 

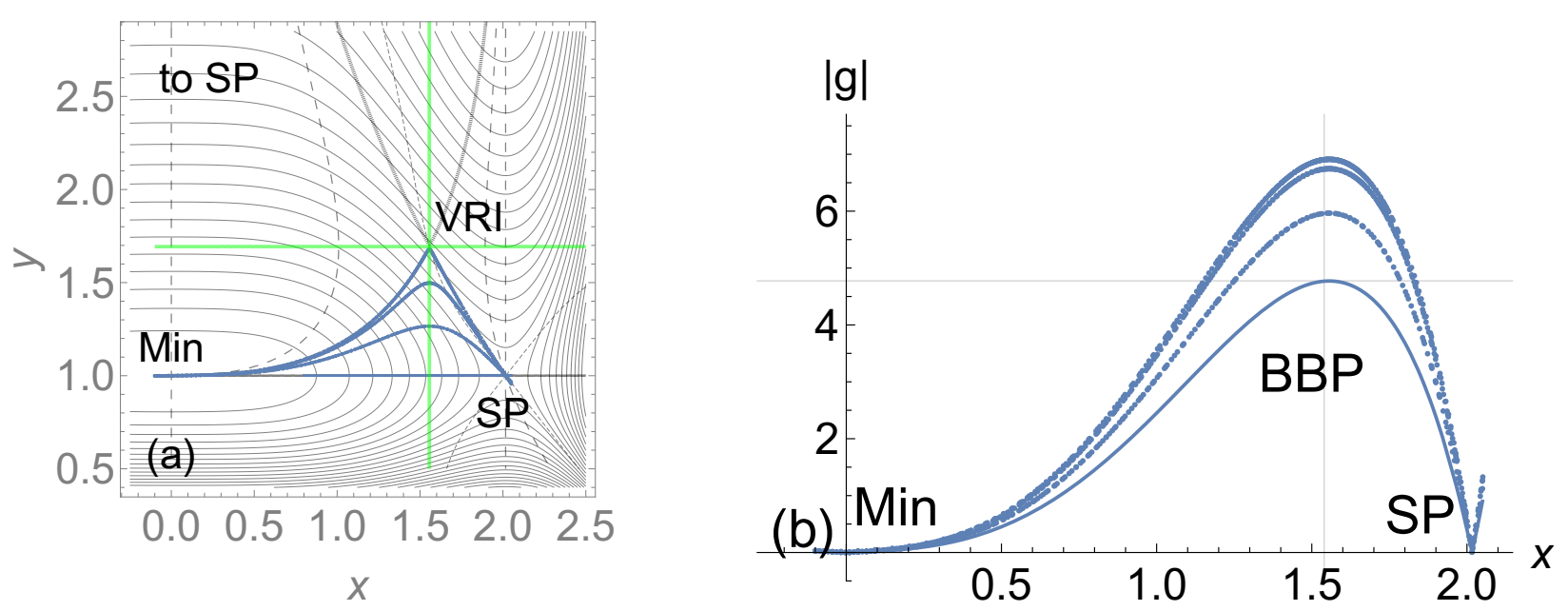

Fig. 4 (a) NTs on a 2D potential. In $x$-direction it is a quartic-sixtic potential, in $y$-direction it is a Morse potential. The blue, bold faced NTs go to the right hand SP of interest, the gray curve is a singular NT through the valley-ridge inflection (VRI) point, and the two dashed curves into the North direction go to the higher SP in $y$-direction. The green cross of lines is the condition $\operatorname{Det}(\mathbf{H})=0$. (b) profiles of $|\mathbf{g}|$ over the blue NTs of panel (a). All BBPs are here on a straight line, where $\operatorname{Det}(\mathbf{H})=0$ holds, at $x=1.54$. Order: the lower curve is the one over MEP, the upper is the one over the singular NT through the VRI point. The NT along the $x$-axis is the optimal pulling direction.

different steepness of the PES in Fig.5, we use different distances between the level lines: below, the distance is 4 units (9 lines), then 10 steps follow with a step of 10 units, and the outer shell of level lines has steps of 25 units (12 lines). The last shown level is at 450 units. Shown are also the condition BBP with $\operatorname{Det}(\mathbf{H})=0$ by a green curve, as well as the convexity border of the surface by a thin dashed curve. If an NT crosses this border then it has a turning point (TP) seen for the energy. For a pulling task, TPs have generally no direct meaning. (An exception is discussed in the example 8.) Many chemical examples are discussed in Ref.[45] where the curve of FDSPs goes through the ridge region of the SP. That means that there a TP exists.

We are interested in a reaction from the lower minimum at $(-1,-1)$ over the SP. All NTs inside the region between the four border points: the two VRIs, the lower Min, and the SP, are allowed NTs for a model mechanochemical reaction. The corresponding pulling direction, $\mathbf{l}$, is the search direction of the special NT which represents it. If

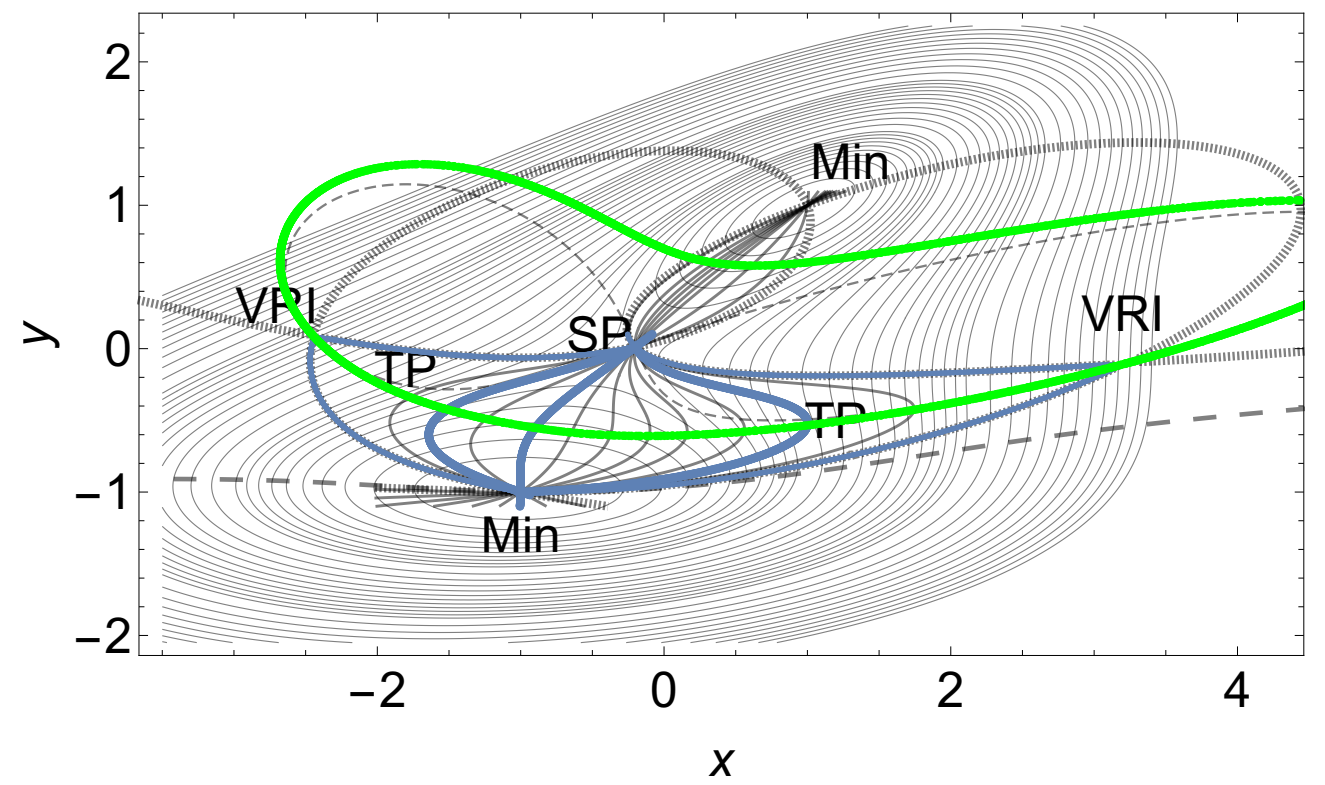

Fig. 5 NTs on a 2D skew quartic potential. Full NTs go to the SP, fat curves are singular NTs through the VRI points. One dashed NT goes into the mountains in $x$-direction. The blue NTs are used for gradient profiles, see Fig.6. Some NTs have turning points (TP). The green curve is the BBP condition. 

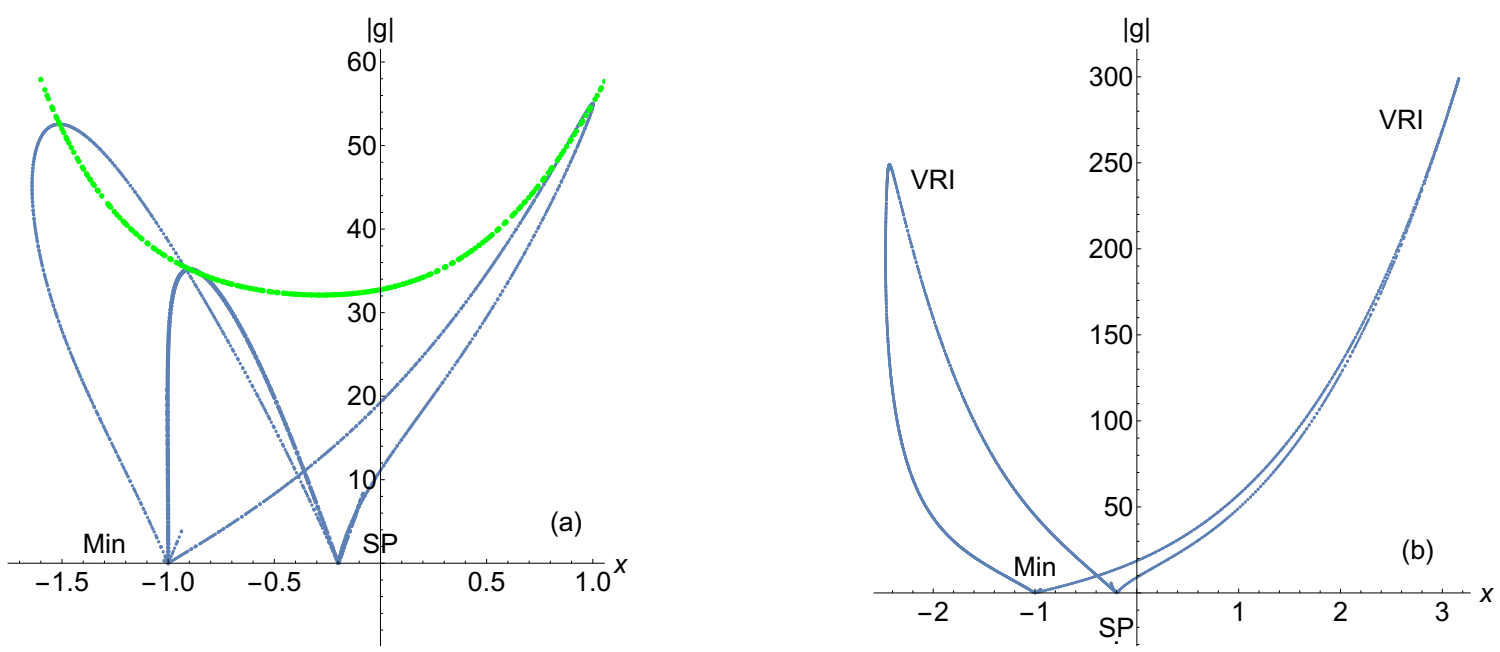

Fig. 6 (a) profiles of $|\mathbf{g}|$ over the three inner blue NTs from the lower minimum to the SP of Fig.5. The BBPs are on the top of the curves (in green). The green curve has a minimum for the optimal NT, compare Fig.7. (b) profiles over the two singular NTs through the VRI points.

$F=\max _{N T}|\mathbf{g}|$ one finishes the pulling task at the BBP, the crossing with the green curve. The regions before the two VRIs are only formally allowed for pulling acts: they need such a high force that they are forbidden (or questionable, at least). 'Other mechanically induced bond rupture processes will likely take place' [40]. In Fig.6 we represent the profiles of the norm of the gradients along five different NTs. The left panel (a) shows the cases along the three inner NTs, the right panel (b) along the two singular NTs. It is clear that there an optimal NT exists where the force, $F=\max _{N T}|\mathbf{g}|$, is minimal, see Section 3. In Fig.7 we show this case where a regular NT and the GE cross the $\operatorname{Det}(\mathbf{H})=0$-line at the same point, being the BBP with lowest $|\mathbf{g}|$ with respect to the others BBPs. It is the optimal NT for a pulling process.

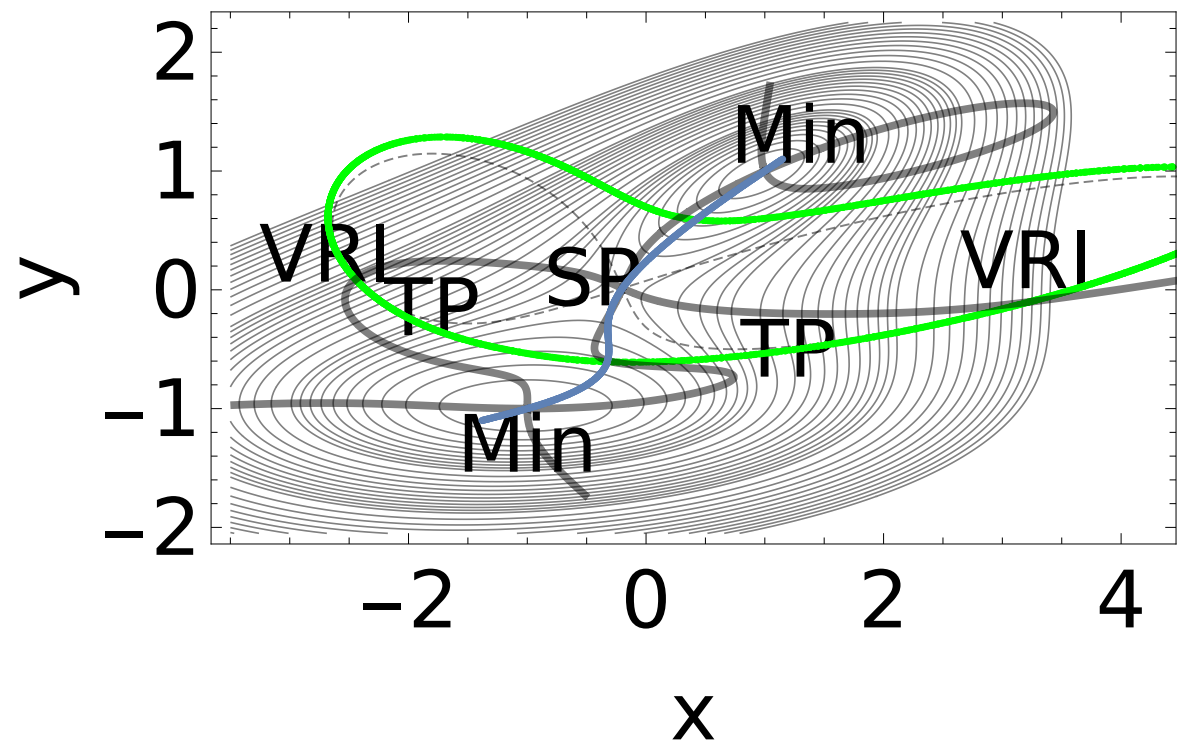

Fig. 7 A special regular NT (blue) and a GE (thick black) cross the $\operatorname{Det}(\mathbf{H})=0$-line (green) at the same point. This point is the BBP with lowest $|\mathbf{g}|$ with respect to the others NTs crossing the green line. The regular NT indicates the optimal pulling direction. 
Example 5

The next example, Fig.8, concerns two quasi 'parallel' SP cols in $x$-direction, thus two reactions compete starting at the same minimum. A reaction can occur along one of two RPs on the PES. The example PES is by Konda et al. [40] being a two-dimensional reduction of a full PES to discuss a ring opening of 1,3-cyclohexadiene. A similar picture is the case of Fig.3A of Ref.[32]. The formula is given in Appendix (A5). The first $S P_{l}$ is slightly lower in energy than the other $S P_{u}$. Again we have chosen five NTs (blue) for the representation of the profile curves of $|\mathbf{g}|$ in Fig.8(b). Also shown is (in green) the profile of the function $|\mathbf{g}|$ over the left $\operatorname{Det}(\mathbf{H})=0$-line. Interesting is the behavior of the profile over the singular NT. The branch on the $x$-axis has its $|\mathbf{g}|$-maximum on the left line $\operatorname{Det}(\mathbf{H})=0$. Here the first eigenvalue of the Hessian is zero; it is the eigenvalue to the eigenvector in $x$-direction. In contrast, the right line $\operatorname{Det}(\mathbf{H})=0$ crosses the $x$-axis at the VRI point. Here the $|\mathbf{g}|$-profile over the $x$-axis does not have an extreme value as can be observed in Fig.8(b). The determinant of the Hessian is the product of all eigenvalues of the Hessian. In the VRI case, the second eigenvalue goes through zero: an intrinsic property of the VRI point. The minimum-valley inflects to the maximum-ridge. The zero of $\operatorname{Det}(\mathbf{H})$ concerns here the two other branches of the singular NT which connect the two saddle points of index one. On these two branches, the $|\mathbf{g}|$-profile from $\mathrm{SP}_{l}$ over the VRI point to $\mathrm{SP}_{u}$ has a maximum at the VRI point. (This is not shown in Fig.8.)
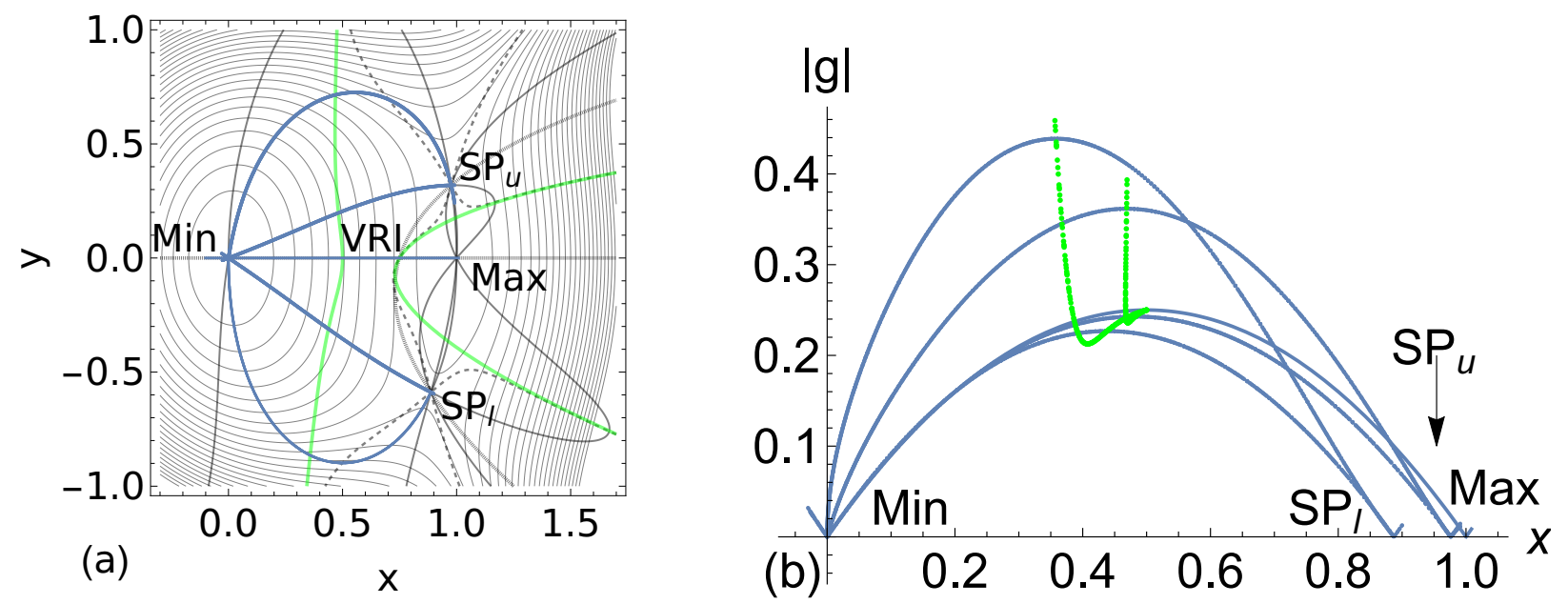

Fig. 8 (a) NTs (black or blue) on the KAM potential [40]. Some NTs to the two SPs have TPs. The five blue parts of some NTs from the minimum to the two SPs and the maximum through the VRI point are used in the right part, two for every SP respectively, and the thin blue one goes to the maximum. The green curves are the condition $\operatorname{Det}(\mathbf{H})=0$. Its crossing with an NT marks the BBP. (b) Profiles of $|\mathbf{g}|$ over the five blue NTs from minimum to the two SPs and the maximum. The curves are represented over their $x$-coordinate. The true pulling BBPs are on top of the curves where the profile over the left BBP condition is presented in green. The $\mathrm{BBP}$ of the $\mathrm{NT}_{\text {low }}$ is somewhat lower than the $\mathrm{BBP}$ of the $\mathrm{NT}_{u p}$.

The two NTs which start at the minimum and meet 'directly' the two SPs later cross the maximum, and still later the other corresponding SP. There is no 'instability' along the pulling lines represented by these NTs, within the meaning of Fig.1(d) of Ref.[40]. Our example contradicts Fig.1(d) of Ref.[40]. We find that the relations are complicated. Only one special pulling force direction along the singular NT corresponds to Fig. 1(d) of Ref.[40]. However this is, as the name suggests, a single direction which may be difficult to meet.

The 'direct' $\mathrm{NT}_{u p}$ from $\mathrm{SP}_{u p}$ downhill to the minimum, for example, leads with the corresponding pulling direction downhill to the BBP of this NT at the crossing of the left $\operatorname{Det}(\mathbf{H})=0$-line. However, the corresponding $\mathrm{NT}_{u p}$ has a continuation after the $\mathrm{SP}_{u p}$ in the contrary direction. It leads to the maximum, and then to the $\mathrm{SP}_{\text {low }}$. The pulling force, say $\mathbf{f}_{u p}$, which drives the $\mathrm{SP}_{u p}$ downhill, at the same time, then drives the $\mathrm{SP}_{\text {low }}$ uphill to the maximum, and then the $\mathrm{SP}_{\text {low }}$ disappears: already a moderate pulling force obliterates this second product valley. The process is shown in Figs.9 and 10 for four values $F=\{0.066,0.133,0.2,0.248\}$ of the pulling force along $\mathbf{l}_{u p}=(0.99,0.141)^{T}$. The schematic sequence of effective PESs follows an analogous representation of Ref.[32].

The direction of the pulling force, $\mathbf{f}_{u p}$, decides that we get the BBP of the NT $\mathrm{N}_{u p}$. Contrary, another direction, say the pulling force, $\mathbf{f}_{\text {low }}$, with $\mathbf{l}_{\text {low }}=(0.977,-0.213)^{T}$ then decides that we get the BBP of the $\mathrm{NT}_{\text {low }}$. Thus we find an analogous behavior for the 'direct' $\mathrm{NT}$ from the lower $\mathrm{SP}_{\text {low }}$ to the minimum. It causes the upper reaction valley to disappear (not shown). The pulling direction decides which reaction path on the PES is used. The optimal 

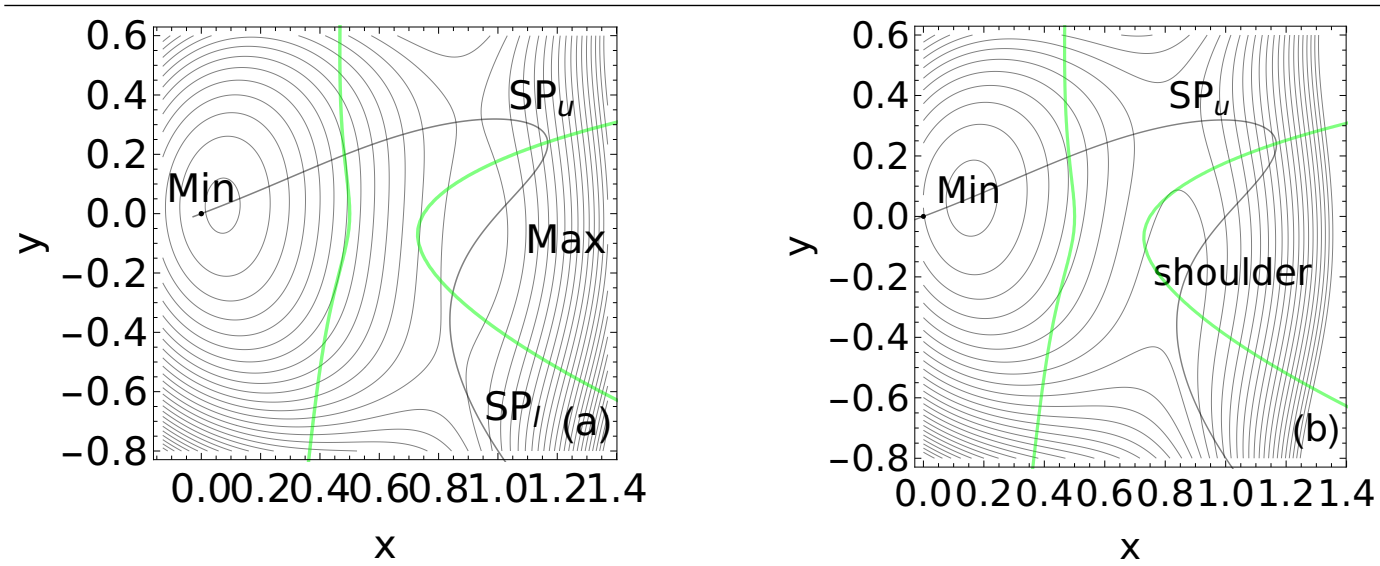

Fig. 9 Two cases of $V_{e f f}$ for two pulling forces of $\mathbf{f}_{u p}$. The saddle $\mathrm{SP}_{l o w}$ disappears on the right hand panel (b). Included are the $\mathrm{NT}_{u p}$ (thin black) for comparison and the lines $\operatorname{Det}(\mathbf{H})=0$ (green) which survive all linear pullings.
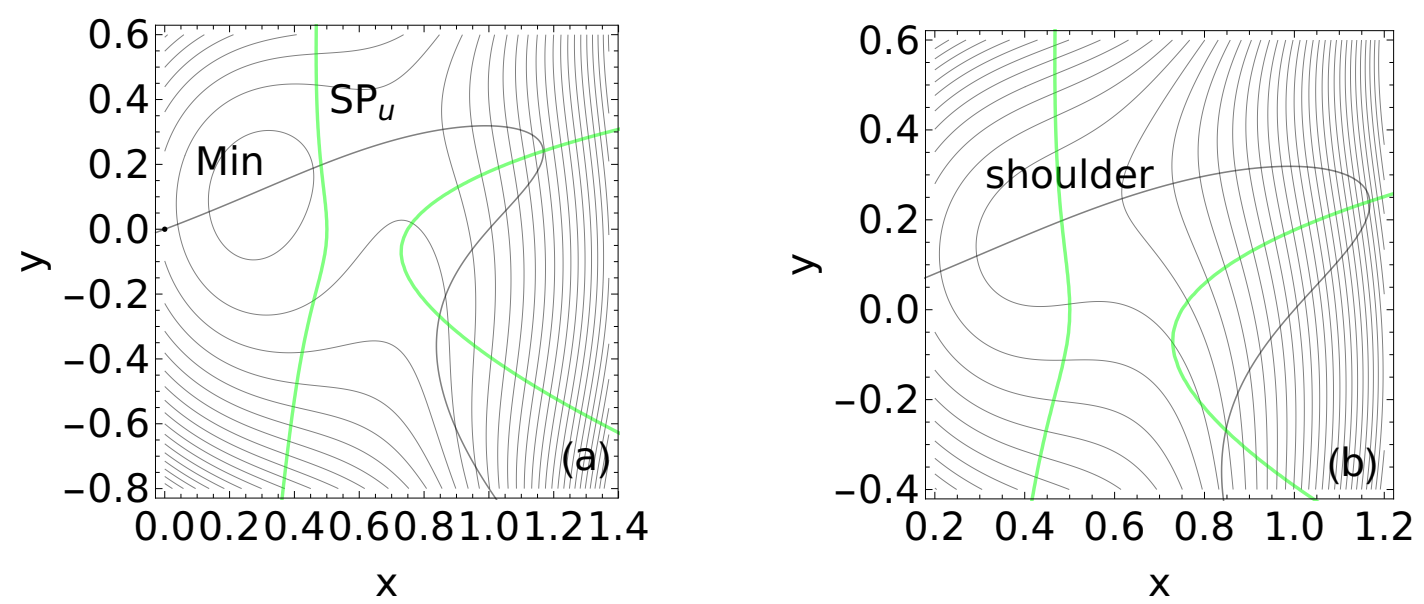

Fig. 10 Two next cases of $V_{\text {eff }}$ for two further pulling forces of $\mathbf{f}_{u p}$. The stationary points, $\mathrm{SP}_{u p}$ and the minimum, move along the $\mathrm{NT}_{u p}$. At least, a shoulder emerges. The former $\mathrm{SP}_{u p}$ and the minimum also disappeare. The left line $\operatorname{Det}(\mathbf{H})=0$ (green) indicates the final $\mathrm{BBP}$ on the right panel. There, only the up-reaction is going on. The former channel of the $\mathrm{SP}_{\text {low }}$ disappeared.

pulling forces, $F_{l o w}$ and $F_{u p}$, are nearly equal, in this example, for both channels, see Fig.8(b).

Conclusion:

Competing reaction pathways can be selected by a corresponding pulling direction.

The VRI point makes the decision between the two pathways.

The conclusion is consistent with a calculated molecular example of Bailey and Mosey [24] to the ring opening of 1,3-cyclohexadiene. They obtained that a given force, $\mathbf{f}$, can guide the system along specific reaction pathways. Other examples are reported elsewhere $[43,46]$.

The main branch of the singular NT from Min to Max on the PES in Fig. 8 goes along the $x$-axis over a VRI point. This is the consequence of the index theorem [37]. The maximum is a saddle of index two which cannot be connected by a regular NT to the minimum with index zero. The VRI point separates the two channels of the two saddles of index one. Now, if one exactly uses the direction of this singular NT for a pulling process, one gets first, for the right green BBP condition, the coincidence of the two SPs and the maximum at the former VRI point into a so-called cusp catastrophe, in the language of catastrophe theory [38,39]. In the theory of PESs its name is monkey saddle $[39,47,48]$. The two product valleys from the two SPs, and the reactant valley meet at one point on $V_{e f f}$. (The two product valleys take up the legs, and the reactant valley takes up the tail of the monkey which can sit on this SP.) The case is schematically shown in Fig. 1(d) of Ref.[40] where is given a chemical example. We show it in Fig.11(a). Later, for stronger $F$, the minimum and the former monkey coalesce to a shoulder at the left BBP. The VRI always survives the linear transformation of a $V_{\text {eff }}$ at its place. At the end, the valley downhill through the shoulder bifurcates into two side valleys and one ridge at the VRI point. All paths go downhill. 


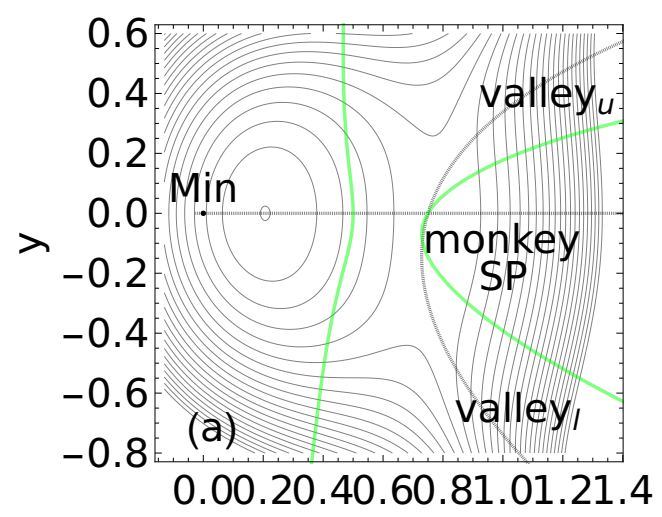

$\mathrm{X}$

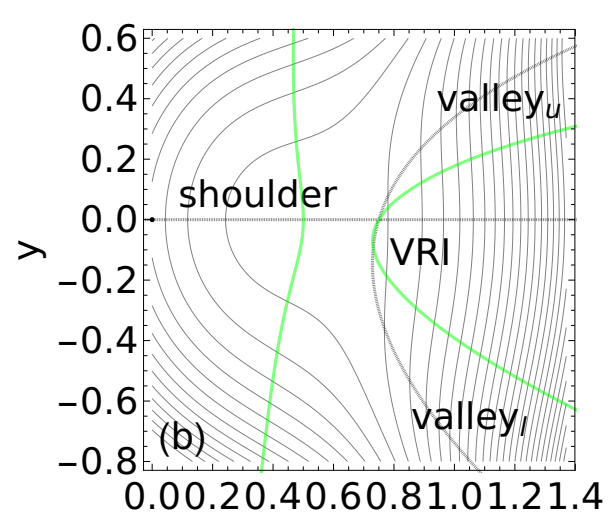

$\mathrm{X}$

Fig. 11 Two cases of $V_{\text {eff }}$ for two pulling forces of $\mathbf{l}_{b i f}=(1,0)^{T}$. (a) the three stationary points, $\mathrm{SP}_{u p}, \mathrm{SP}_{l o w}$ and Max, move along their branches of the $\mathrm{NT}_{b i f}$. At $F=0.164$, it emerges a monkey saddle at the VRI point. The former slope here has flattened out to an SP-plateau. The former minimum still exists. (b) at $F=0.25$ the left line $\operatorname{Det}(\mathbf{H})=0$ (green) indicates the final BBP where the minimum disappears. The slope at the VRI point is turned around, now downhill.

Note that the two SPs, in this case, are not symmetric. Nevertheless, there exists the singular NT whose side branches connect the two saddles over the VRI point. It holds by the index theory [37]. A pulling along the direction of the singular NT enforces the cusp catastrophe. It takes place also in nonsymmetric cases, compare a more special conjecture in Ref.[40].

Example 6

This example, Fig.12, concerns again two 'parallel' SPs in $x$-direction, thus two reactions compete, however, now they start at two different minimums. The $\mathrm{SP}_{3}$ may be on the reaction path of an isomerization of a molecule, where the two other parallel Morse pathways with the $\mathrm{SP}_{1}$ and $\mathrm{SP}_{2}$, correspondingly, may describe the dissociation coordinate of a part of the molecule. Every way starts at its own isomer: $\mathrm{Min}_{1}$, or $\mathrm{Min}_{2}$. The kind of problem is prepared by Fig. 3 (B) of a paper by Suzuki and Dudko [32] where also a chemical background is reported.
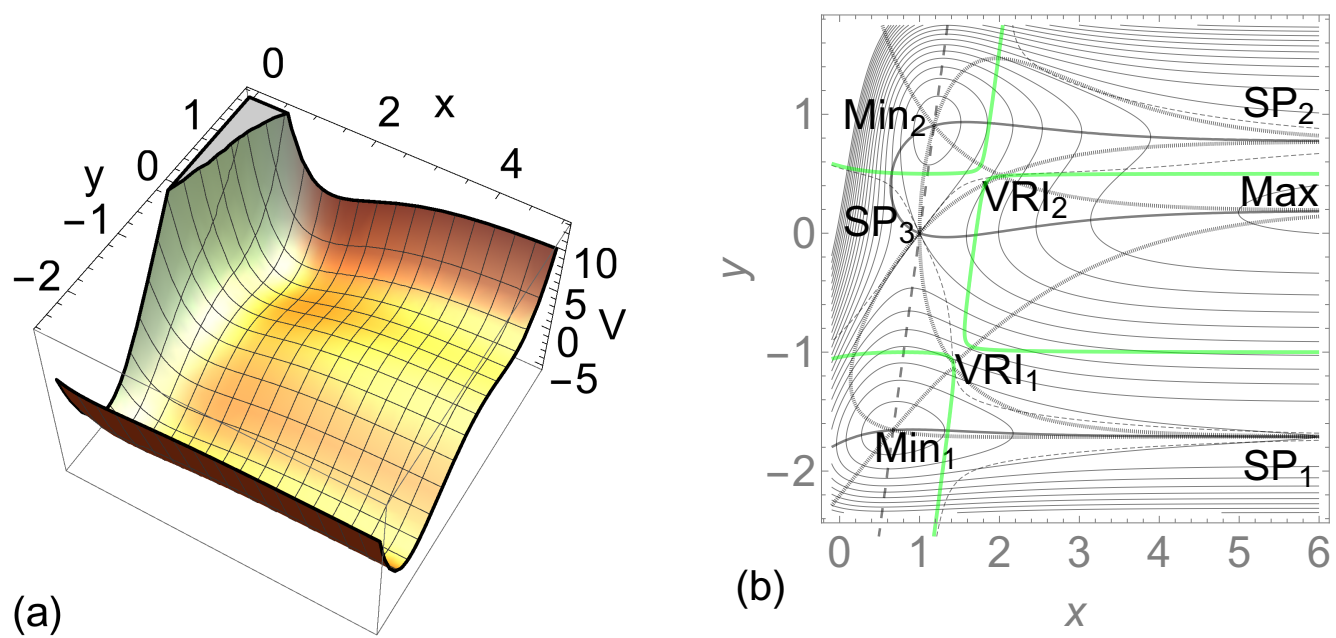

Fig. 12 (a) A potential with two minimums and two escape reactions. (b) NTs on the same surface. The black NT is in the $x$-direction, the bold dashed NT is in the $y$-direction, and the gray curves are singular NTs which bifurcate at the VRI points. The green curves describe the condition $\operatorname{Det}(\mathbf{H})=0$. Their crossing with an NT mark the BBP.

A similar PES is discussed in Ref.[28] but with two orthogonal dissociation pathways. For that problem, one can imagine in Fig. 12 the valley 1 turned by $-45^{\circ}$ and the valley 2 turned by $+45^{\circ}$.

We can also relate the example to the well-known Curtin-Hammett/Winstein-Holness [49] (CHWH) kinetic system. A PES reported in Fig.12 is associated to a reaction mechanism which kinetic equations are just of the type 
$\mathrm{CHWH}$. If the potential energy of $\mathrm{SP}_{3}$ is lower than the potential energy of both, $\mathrm{SP}_{1}$ and $\mathrm{SP}_{2}$, the kinetic behavior is just CHWH. In the opposite situation, when the $\mathrm{SP}_{3}$ is higher then the potential energy of both, $\mathrm{SP}_{1}$ and $\mathrm{SP}_{2}$, we have an anti-CHWH type. No statement can be made when the $\mathrm{SP}_{3}$ is close to the potential energy of both, $\mathrm{SP}_{1}$ and $\mathrm{SP}_{2}$. In any case the study of the BBPs and the corresponding mechanochemistry force gives us the possibility to change the kinetic behavior of a Curtin-Hammett/Winstein-Holness kinetic system, which is very desirable in many chemical situations.

The formula for example 6 is given in Appendix (A6). Here, the first $S P_{1}$ is lower in energy than the $S P_{2}$, but the isomerization $S P_{3}$ is in between. $\mathrm{Min}_{2}$ is higher than $\operatorname{Min}_{1}$. At $V R I_{1}$, for example, the convex valley lines around the minimum $\mathrm{Min}_{1}$ end and a ridge begins to the maximum at the right hand side. The pathway $\operatorname{Min}_{1}$ to $V R I_{1}$ to Max is ascenting. The valley path from the minimum bifurcates at the $V R I_{1}$ into two side branches to the two SPs, $S P_{1}$ and $S P_{3}$.

A first pulling possibility will be the enforced isomerization: a pulling force along one of the $y$-family of NTs will overturn one of the two minimums, see Fig.13. Then the 'usual' chemistry will take place under corresponding thermal excitation, thus the one open dissociation channel will be used. In the sum, the reaction is driven into the favored direction. A mechanochemical switch, may be of this kind, is described elsewhere [27,43, 46].
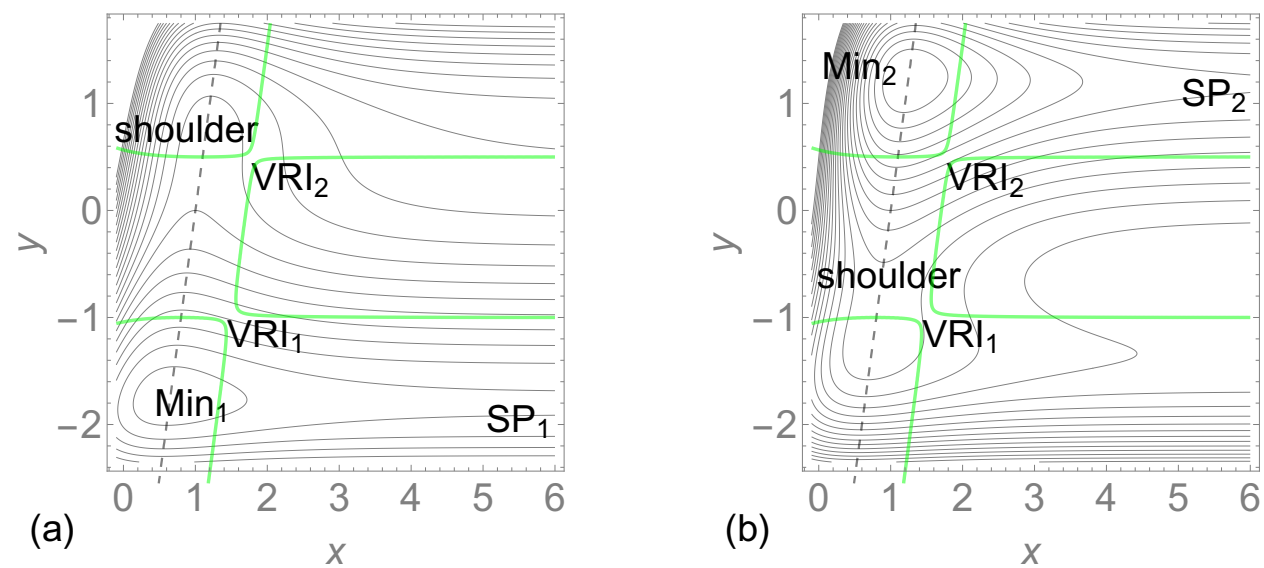

Fig. 13 (a) effective potential where minimum $\mathrm{Min}_{2}$ is overturned. The direction is $\mathbf{l}=(0,-1)^{T}$, and the force is $F=1.875$. $\mathrm{Min}_{2}$ and $S P_{3}$ coalesce to a shoulder. $S P_{2}$ disappeared. (b) effective potential where minimum Min 1 is overturned. The direction is the contrary $\mathbf{l}=(0,1)^{T}$, and $F=4.75 . \mathrm{Min}_{1}$ and $S P_{3}$ coalesce to a shoulder.

We will discuss the new question: can we find a mechanochemical scenario to connect complementary reaction ways, from $\mathrm{Min}_{1}$ to $\mathrm{SP}_{2}$, or vice versa, from $\mathrm{Min}_{2}$ to $\mathrm{SP}_{1}$ ? The answer is: yes; however, it has no practical relevance. This second possibility is opened, theoretically, by a known property of NTs. The VRIs are the border points for the different families of NTs which connect the minimums with the different SPs in their direct neighborhood [44]. Thus, no regular NT can connect the $\mathrm{Min}_{1}$ with the $S P_{2}$, or the $\mathrm{Min}_{2}$ with the $S P_{1}$. However, in both cases there is exactly one NT, the singular NT through the corresponding VRI point, which can open such a connection. If a pulling scenario is found exactly in one of the directions of the singular NTs, one could go crosswise. In Fig. 14 we show these two cases. The direction is given by the singular NT (gray) with $\mathbf{l}=(0.247,0.969)^{T}$, and magnitude $F=8.0$ in the left panel. $S P_{3}$, Max and $S P_{1}$ coalesce first to a monkey saddle at the $\mathrm{VRI}_{1}$ (not shown), and Min 2 and $S P_{2}$ later coalesce to the shown shoulder. At the same $F$-interval, the monkey flattens to a 'usual' VRI, however, now all four branches of the singular NT descending beginning from bottom left. For the right panel (b), the direction belongs with $\mathbf{l}=(0.734,-0.679)^{T}$ to the NT (gray) through $\mathrm{VRI}_{2}$. Early in the pulling, for $F=1.25$, the $M_{i n}$ and the $S P_{1}$ coalesce to a shoulder (not shown). Later, $S P_{3}$, Max and $S P_{2}$ coalesce to a monkey saddle at $F=2.25$ at the position of the $V R I_{2}$, this effective PES is shown. The former slope of the PES at the $V R I_{2}$ is flatten out to an SP-plateau. It is exactly the meaning of the monkey saddle. 3 times more level lines to illustrate the flat remaining Min 2 are used here. The final BBP is at $F=2.75$ where the monkey saddle and the $\mathrm{Min}_{2}$ coalesce, and they form again a usual shoulder (not shown); the place is the crossing of the singular NT with the left upper line $\operatorname{Det}(\mathbf{H})=0$.

Conclusion:

Again, competing reaction pathways can be selected by a corresponding pulling direction.

The VRI points make the decision between the pathways. 

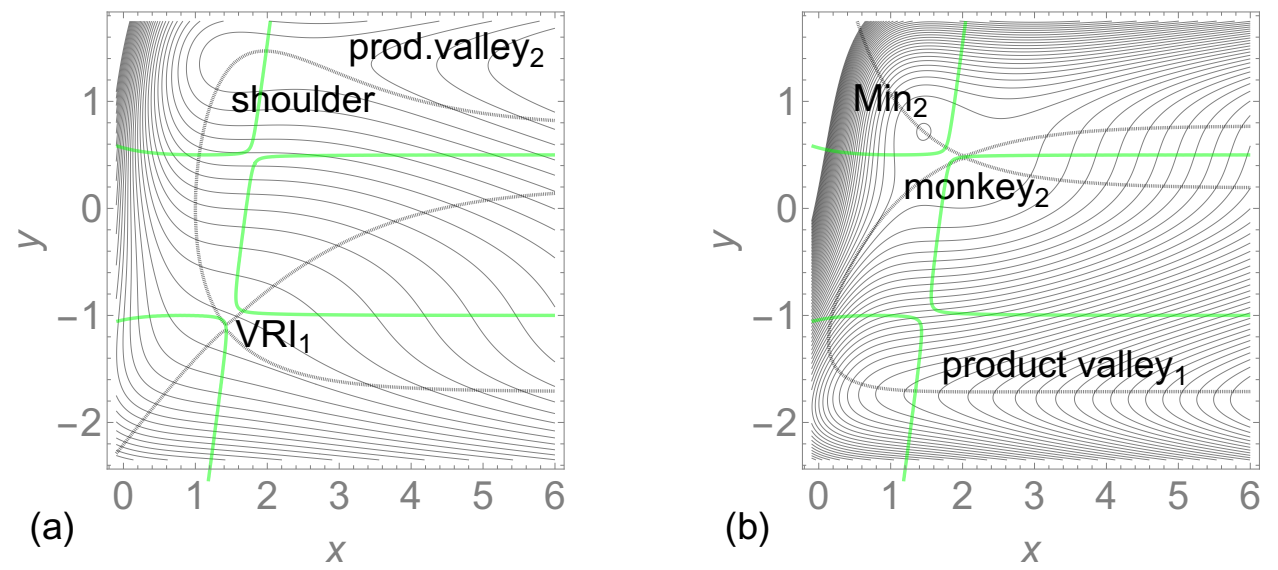

Fig. 14 (a) effective potential where minimum $\mathrm{Min}_{1}$ is overturned and reaction 2 is enforced, by a direct pulling process along the singular NT (gray). (b) effective potential where minimum $\mathrm{Min}_{2}$ is overturned, see text. The singular NT is again included.
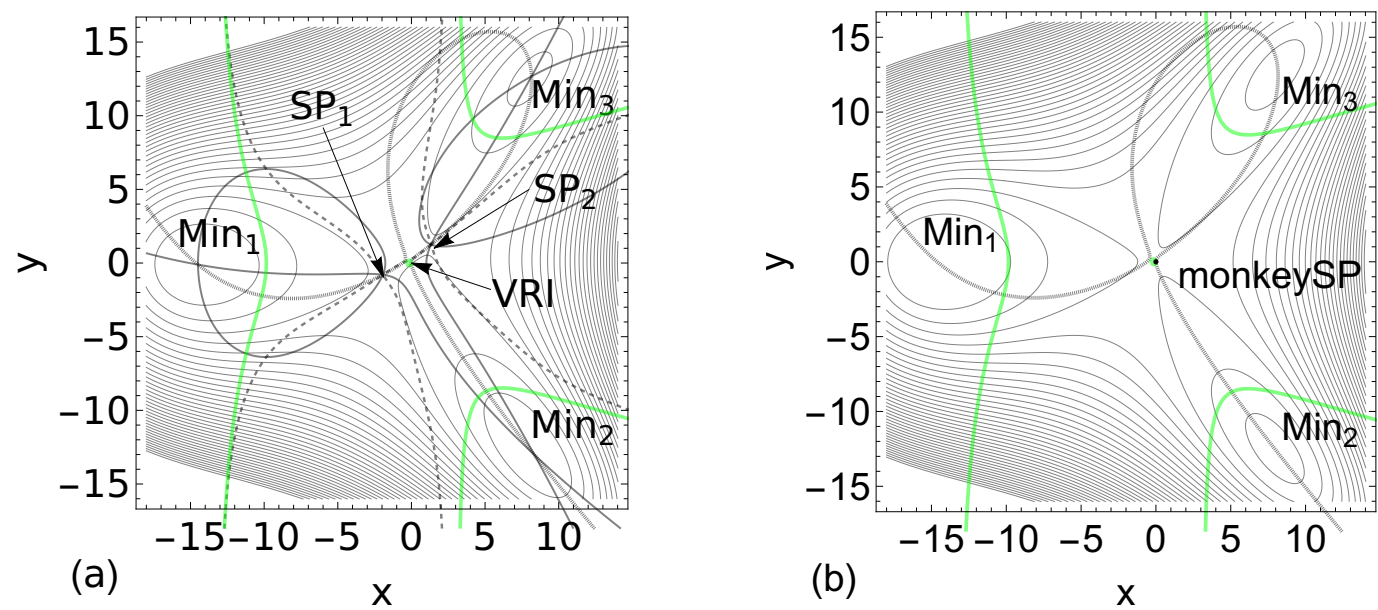

Fig. 15 (a) NTs (gray) on the BQC potential [41]. There are three minimums, two saddles, and one VRI point. The PES is associated to a reaction $\operatorname{Min}_{1} \rightarrow \operatorname{Min}_{2}+\operatorname{Min}_{3}$. The mechanism for this reaction consists of two elementary reactions discriminated by the VRI point. Each product is associated with different minimums of the PES. Green curves are the condition $\operatorname{Det}(\mathbf{H})=0$. Their crossing with an NT mark a BBP. (b) pulling along the singular NT with $F=4.125$ and in direction $\mathbf{l}=(0.643,-0.766)^{T}$, see text. A monkey saddle emerges.

The PES of this example was constructed for the transition from one minimum to two products, however, the reactant valley is finished by only one SP [41], see Fig.15. The VRI point is in back of the saddle of the reactant. The formula of the PES is given in Appendix (A7). An analogous PES is treated elsewhere [50]. A summit does not exist here, an SP of index two. The SPs of index one are crossed by three ridges, depicted by the small dashed convexity border of the level lines. One hypothesizes a PES such that a flat intermediate region has one entrance and two exits. It is quite unlikely that the entrance will be dynamically coupled with equal strength to the two exits [41]. We associate this dynamical situation with the mechanism that a reagent A, which belongs to Min 1 , can go to two products. We explain this nonsymmetric rearrangement bifurcation, $\mathrm{A} \rightarrow \mathrm{B}$ and $\mathrm{A} \rightarrow \mathrm{C}$, in terms of the PES model: in between the two SPs of the entrance to this region there exists a nonsymmetric bifurcation. However, here it is not a monkey SP.

The SPs are near the VRI point, and the $\mathrm{SP}_{1}$ is slightly higher in energy units than the VRI point, and this point is slightly higher than the $\mathrm{SP}_{2}$. The SPs may be nodes of an RP, however, no direct steepest descent (SD) exists from the left minimum A to the minimum $\mathrm{C}$ top right, $\mathrm{Min}_{3}$. The SD from the two SPs leads to the product $\mathrm{B}$ at the right bottom, Min 2 . (An enlarged part of the central region of the PES is shown in Ref.[41].) Again, we ask for a 
possible pulling scenario on such a model PES: can we find a force direction to enforce one of the two possible partial reactions? The answer is: one and a half times yes.
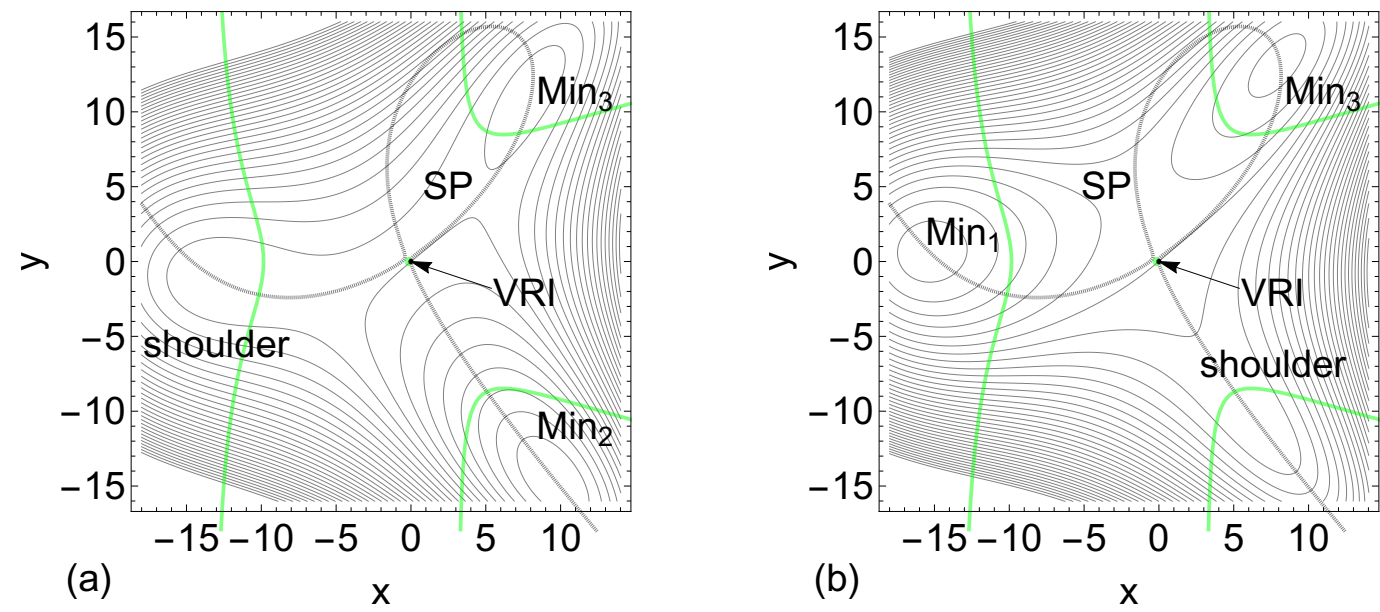

Fig. 16 Effective PESs for pulling along the singular NT (gray) on the BQC potential [41]. (a) to direction $\mathbf{l}=(0.643,-0.766)^{T}$ for force $F=38.9$ units a shoulder emerges between the former reactant minimum, $M_{i n}$, and saddle $S P_{1}$. (b) for the force $F=31$ units with direction $\mathbf{l}=(-0.643,0.766)^{T}$ a shoulder emerges between the former $\operatorname{Min}_{2}$, and the former monkey saddle of Fig.15(b). Green BBP curves and the VRI remain.

The thick gray curve in Fig.15 is the singular NT which connects all three minimums over the two SPs, and which bifurcates at the VRI point. (It is repeated in the next figures.) There is no regular NT from the Min 1 to the Min 3 . To connect the Min 1 and the $\mathrm{Min}_{3}$, we only have at hand the singular NT. The direction of the gradient at every point on this singular NT, $\pm(0.643,-0.766)^{T}$, is applied to a pulling. Fig.16 shows the result for two different forces, $F=\{38.9,31\}$. If the force is small, and $\mathbf{l}=(0.643,-0.766)^{T}$, the two SPs and the VRI coalesce to a monkey saddle, see Fig.15(b). Note that the functional condition $\operatorname{Det}(\mathbf{H})=0$ has an isolated solution at the VRI point. In this case, the two reactions, $\mathrm{A} \rightarrow \mathrm{B}$ and $\mathrm{A} \rightarrow \mathrm{C}$, seem to be equivalently possible.

For a stronger force, $F=31$ units, see Fig.16(b), we get a main valley from reactant to product C, the former $\mathrm{Min}_{3}$. The $\mathrm{Min}_{2}$ disappeared. However, we cannot overturn the reactant minimum, in this case. We have only one remaining valley between the reactant, and one selected product, and it remains an SP in between. Here the unsymmetry of the zero-force PES plays an important role, as well as the loop of the NT in the right upper corner of the figures.

In the contrary case, for a force, $F=38.9$ units into direction $(0.643,-0.766)^{T}$, the reactant minimum, Min $_{1}$, and the saddle $S P_{1}$ coalesce to a shoulder: one main valley remains between the two products. Because the $\mathrm{Min}_{2}$ is much deeper on this effective PES, one can assume that this product is enforced by the pulling, see Fig.16(a). A further increase to $F=59.95$ would finally lead to a shoulder in the upper part of the PES (not shown).

Because we have only the singular NT to connect all three minimums, there is only one pulling (theoretically, with \pm ) which can connect all three minimums. But there are only two possibilities for the direction. It can be positive or negative: note that we do not discuss here the technical possibility of such a pulling, or a compression [51]. The values being large enough in one direction overturn the $\mathrm{Min}_{1}$ for a chemical reaction A $\rightarrow$ B in Fig.16(a). The values being large enough in the other direction overturn the $\mathrm{Min}_{2}$ for a chemical equilibrium $\mathrm{A} \leftrightarrow \mathrm{C}$, however, then the structure of the double-minimum valley remains on the effective PES up to very high, unrealistic forces, $F$. No possibility exists to enforce an overturn of $\mathrm{Min}_{1}$ for an enforced chemical reaction A $\rightarrow$ C in Fig.16(b). Note that all stationary points of the new effective PESs move on the singular NT of the zero-force PES, because this NT is used for the pulling direction, and it is also a singular NT on the effective PESs.

\section{Example 8}

The PES of this example was constructed for the transition pathway from minimum $\mathrm{C} 7_{e q}$ to minimum $\mathrm{C} 7_{a x}$ of the alanine dipeptide molecule [52]. Originally, the two coordinates are two intrinsic dihedral angles. Other coordinates are like spectators; they are projected out of the 2-dimensional treatment. The modified NFK-PES [37,44] in Fig.17 is an interesting example: there does not exist an NT without a TP from the minimum to the SP. On the NFK PES 

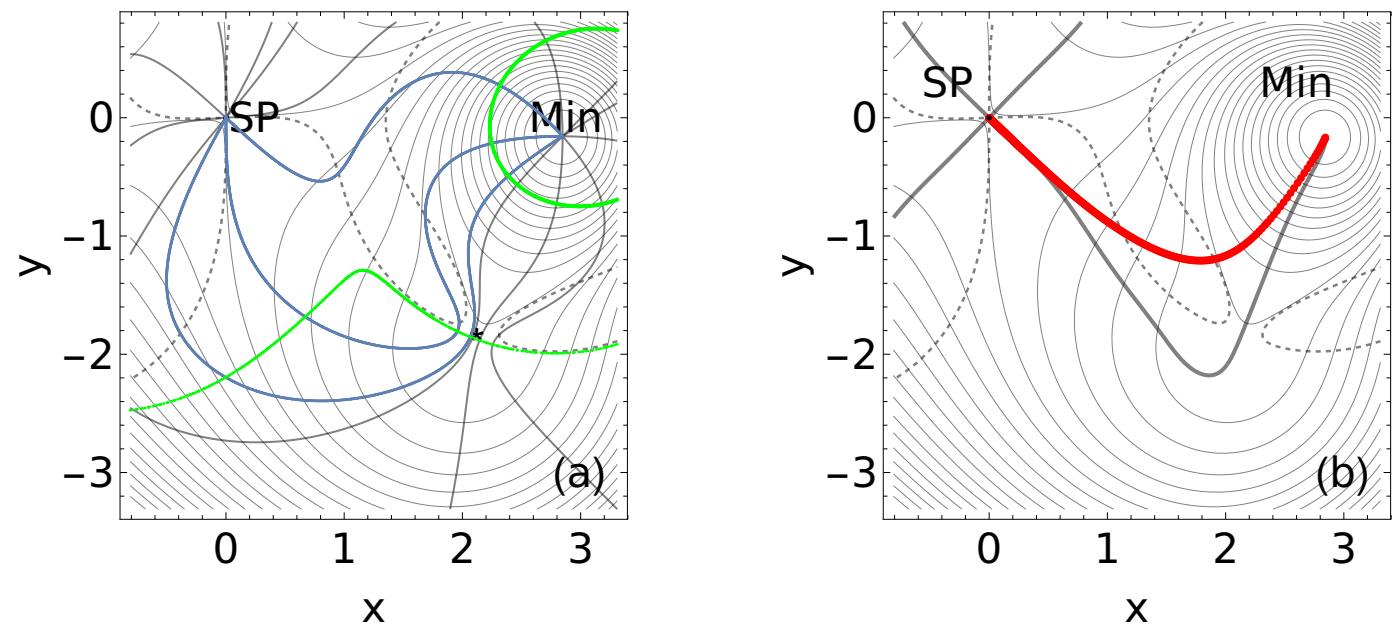

Fig. 17 (a) NTs (black or blue) on a modified NFK potential. Three blue parts from the minimum to the SP are used in the next Fig.18. Green curves are the condition $\operatorname{Det}(\mathbf{H})=0$. Its crossing with an NT marks a BBP. (b) The bold faced curve is the GE, here it represents the RP, the so called MEP, from the minimum to the SP. On the other hand, the steepest descent from the SP (red curve) goes over the nose. It is not the MEP.

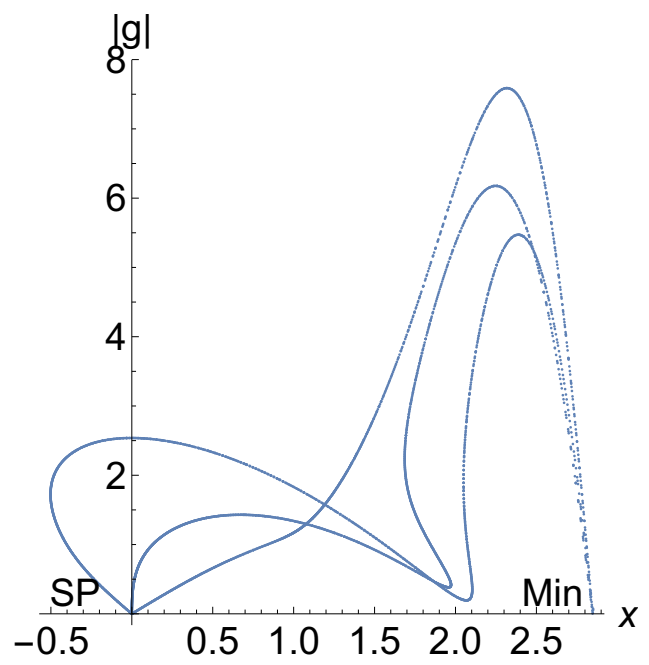

Fig. 18 Profiles of $|\mathbf{g}|$ over the three blue NTs from minimum to SP of Fig.17(a). The curves are represented over the $x$ coordinate. The true pulling BBPs are on the right top of the curves. The order goes like in Fig. 17 from the top to the lower curve.

we have TPs with an energy lower than the SP. However, we have seen in the former example that the existence of TPs of NTs does not play a direct role for the pulling problem (from a theoretical point of view). Additionally, in the shown region of the figure does not emerge a VRI point. VRIs have a quite higher energy in regions outside of the figure. For this modified surface, one has constructed at point $\approx(2,-2)$ a quasi shoulder [37,44]. A former small intermediate has disappeared here. From the SP downhill to this shoulder one finds a nose, a ridge, depicted by the small dashed convexity border of the level lines. The nose is between the so-called minimum energy path (MEP) from the SP, and the minimum bowl.

Fig.17(b) shows the gradient extremal (GE) $[41,48,53,54]$ which plays here the role of the MEP, however, the steepest descent from SP $[55,56,57]$ goes across the ridge: so, the surface of this example is an exception.

We find two kinds of NTs: first a sort with one BBP on the inner circle of the green BBP condition around the minimum. This is the usual case, represented by the upper blue NT in the figure, and second a sort with three crossings of the BBP condition, see Figs.17 and 18. The NTs also cross the second curve $\operatorname{Det}(\mathbf{H})=0$. On $|\mathbf{g}|$ are two maximums and one minimum. The one BBP of the former, direct NT also converts to the highest maximums of the two other NTs. 


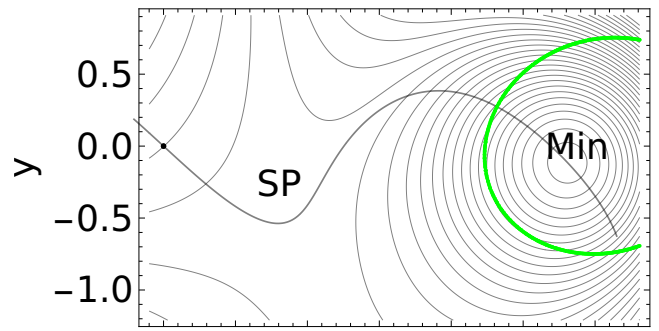

(a)

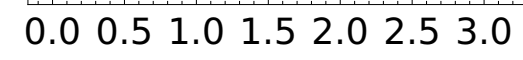

$\mathrm{X}$

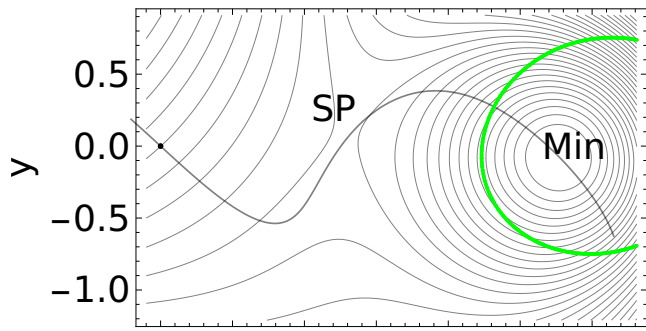

(b) $\quad 0.00 .51 .01 .52 .02 .53 .0$

$\mathrm{X}$

Fig. 19 Two cases of $V_{\text {eff }}$ of the NFK PES for two pulling forces, $F_{1} l$ and $F_{2} l$ : the SP moves downhill along the original NT (black). The effective PES changes, however, the used NT and the circle $\operatorname{Det}(\mathbf{H})=0$ (green) survive all linear pullings.

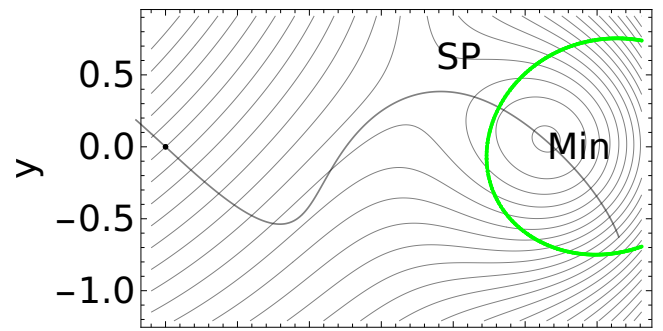

(a)

0.00 .51 .01 .52 .02 .53 .0

$\mathrm{X}$

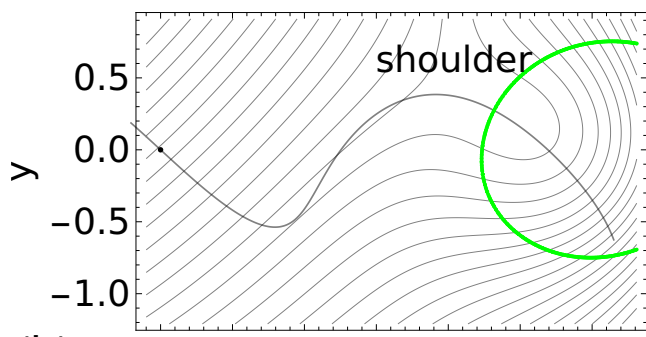

(b) $\quad 0.00 .51 .01 .52 .02 .53 .0$

$\mathrm{X}$

Fig. 20 Two further cases of $V_{e f f}$ for two further pulling forces, $F_{3} \mathbf{l}$ and $F_{4} \mathbf{l}$ of the same force direction l: at least a shoulder emerges where the reaction is enforced. The former SP and the minimum disappear.

The direct NT with direction $\mathbf{l}=(-0.74,0.68)^{T}$ on the PES from the SP downhill, for example, moves this SP with the corresponding pulling force downhill the NT to the BBP of this NT at the crossing of the green border. It is demonstrated in Figs.19 and 20 where the following forces are used: $F=\{1.0,1.9,4.83,7.32\}$.

The NTs with three crossings of the (green) condition $\operatorname{Det}(\mathbf{H})=0$ form a special case what is demonstrated by the series of effective PES in Figs. 21 and 22. First, for a small pulling force, $F_{1}=1.56$, into the direction of the NT with $\mathbf{l}=(-0.87,-0.5)^{T}$, the SP is moved uphill the left ridge of the original PES (but downhill in effective energy). It is shown in Fig.21(a). Additionally to the movement of the SP, an intermediate minimum and a second SP emerge, both also lie on the used NT. If the force increases, $F=2.56$ in the right panel (b), the first SP and the intermediate again disappear in a shoulder, and only one SP survives. In Fig.22 we use the forces $F=\{2.89,5.12\}$. For the final $\mathrm{BBP}$ force, on the green circle around the original minimum, the last SP also disappears and one gets again the final shoulder which enforces the planned reaction. Again, the direction of the pulling force, $\mathbf{f}$, decides which scenario one finds. A set of directions leads directly to the shoulder on the inner green circle of BBPs. Another set of directions goes on over an intermediate, and later to the final BBPs.

Elsewere $[27,58]$ it is discussed which influence an intermediate of the original PES can have on a mechanochemical process. This example 8 shows that a certain pulling force can induce an effective, new intermediate in a certain interval of the force, $F$. Especially the finding that for a low force, $F$, the intermediate is open with a certain reaction rate [27] which disappears near the hard, final force near the BBP, is consistent with our model. The effective PES with an intermediate can be a model PES for the transition from 'catch a bond' to 'slip a bond' under a pulling force [50].

Conclusion: If one finds a second, lower maximum along a given NT on the $|\mathbf{g}|$-profile then one can get an intermediate minimum on $V_{e f f}$ on the pulling path for intermediate forces. However, this minimum will disappear again on the way to the hard BBP of the problem.

Note that the 'roundabout way' along an indirect NT needs here a little lower BBP force, see Fig.18, because the movement of the initial minimum to this BBP goes along the flatter MEP of the original PES, along the eigenvector to the smallest eigenvalue of the minimum. In contrast, the direct NT between Min and SP goes after the Min along the steeper slope along the second eigenvector. 

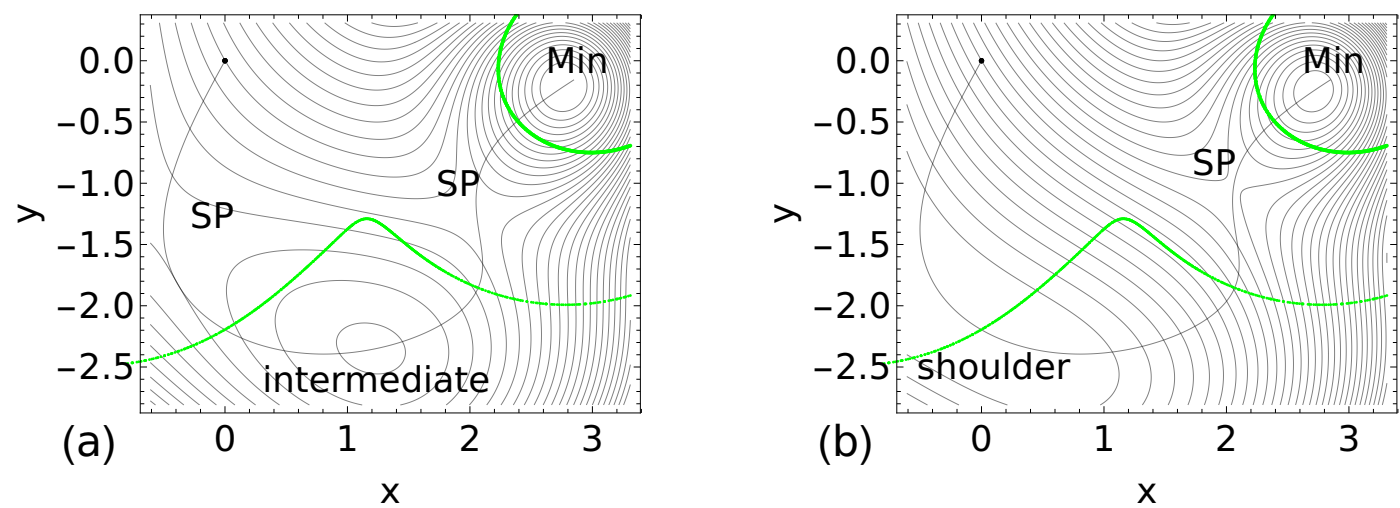

Fig. 21 Two cases of $V_{\text {eff }}$ of the NFK PES for a lower NT (thin black) with two maximums of the $|\mathrm{g}|$-profile, at two pulling forces: first the SP moves uphill along the original NT, but later correctly downhill. (a) a new minimum emerges, again on the given NT. (b) the second green curve marks the emergence of an upper shoulder.
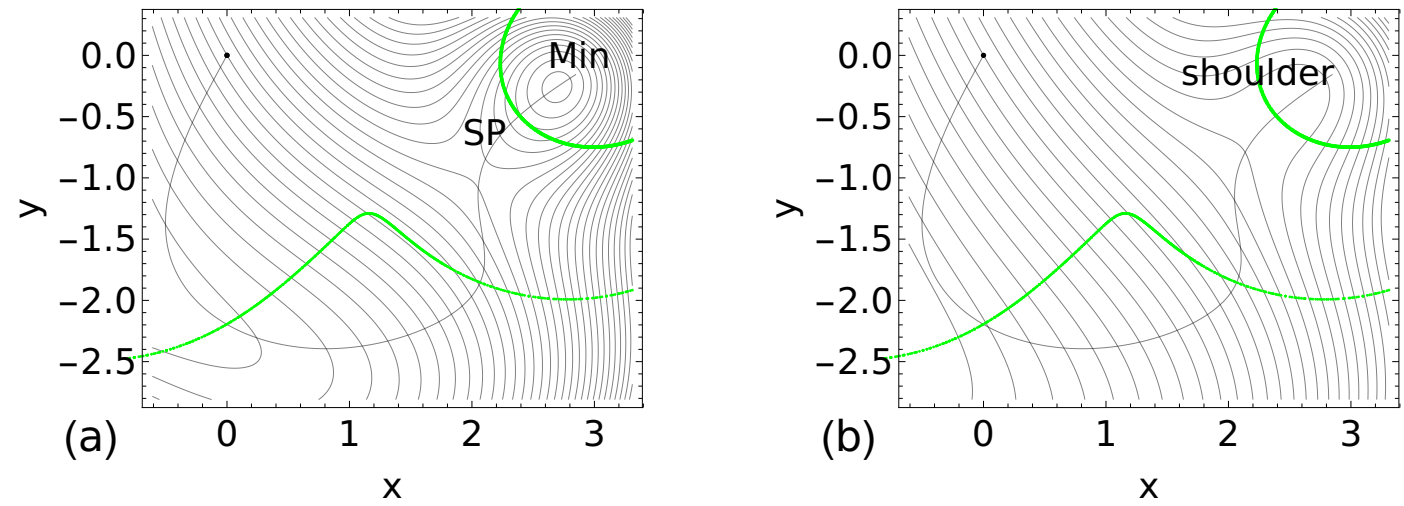

Fig. 22 Two next cases of $V_{\text {eff }}$ for a lower NT (thin black), at the next two further pulling forces of the same force direction l: at least a shoulder emerges where the reaction is enforced. All the former SPs and the minimums disappear.

\section{Discussion}

The NTs on a PES are discriminated by the emergence of VRIs, valley-ridge inflection points. All directions of regular NTs in between the singular NTs to two neighbor VRIs are allowed for a pulling scenario. It also holds in the $N$ dimensional case of the PES, because the VRIs, together with the singular NTs, form manifolds, and these manifolds partition the full configuration space for NTs [4,59]. The VRI points determine the pulling channels [37]. NTs form a family of dense curves over the configuration space. Around every stationary point, into all directions of a possible force, l, starts an NT. The range of all adapted directions for an NT family to a given SP is a range of useful forces of a mechanochemical pulling scenario for the molecule. Changing from one NT-family to another behind a VRI point corresponds to a reaction mechanism switch $[27,40]$. It holds at least from a theoretical point of view. Practical examples demonstrate: another barrier of the $N$-dimensional PES can break down if the pulling force becomes too high. An example is discussed for the molecule 1,3-cyclohexadiene [24] where a ring-flip reaction happens for a pulling force in direction of the conrotatory pathway, a reaction in another dimension than expected in the two-dimensional picture of the treatment.

The calculation of the SPs on many surfaces $V_{\text {eff }}$ is reduced to the curve following along the NT on the original, zero-force $V$ in direction 1, up to the corresponding force $|\mathbf{g}|_{\max }=F$ of the final BBP. To follow an NT in an $N$-dimensional space is a possible task, using Eq.3 and Eq.8, because it is a one-dimensional curve which is easy to follow $[4,8,9]$. The NT-tool works perfectly well in any number of dimensions. Special calculations are done for applications of NTs in Chemistry [60,61,62,63,64,65,66,67]. The search of VRI points by NTs is possible [68,69]. It is especially reported on the PES of alanine dipeptide [70], on the PES of the cyclopropyl radical [71], as well as on the PES associated to the ring opening of the cyclobutene [72]. 
We formulate some simple

Rules for a pulling scenario and the barrier breakdown point (BBP):

(i) All directions of regular NTs between two neighbor VRIs are allowed directions (theoretically) for a pulling. Thus, there is usually a very wide field of possible pulling directions for an enforced reaction by pulling. However, there is an optimal direction where the pulling force has a minimum at its BBP compared with other NTs.

(ii) In practice, it will be of interest that a technical usable pulling direction is an allowed one, and if so, is it near the optimal case?

(iii) For a deep minimum and a flat SP (of 'Sancho-Panza'-type [32,77]) of the zero-force PES, the BBP is 'early' near the minimum.

(iv) For a flat minimum and a steep SP (of 'Don-Quixote'-type [32,77]) of the zero-force PES, the BBP is 'late' near the SP.

(v) Competing reaction pathways which start at the same minimum can be selected by a corresponding pulling direction.

(vi) For two different heights of two competing SPs the final force, $F=|\mathbf{g}|$ max , at the two BBPs may vary in different kind.

(vii) If a pulling force, $F$, needed for a planned reaction, is 'too high', then another BBP anywhere on the N-dimensional PES can take place.

The Eq.1 was already proposed by Thornton [73] in 1967 to predict both the new SP and the reaction path when a molecular system is perturbed due to a change of substituent or solvent. The conclusions of Thornton are very close to the rules explained in the present study. They were derived on the original PES, $V(\mathbf{r})$, being quadratic with respect to $\mathbf{r}$.

All stationary points of an FDSP curve of the different effective PESs are obtained automatically, if one follows the corresponding NT. There is no need for a new calculation of any SP on the different effective potentials which was already remarked by Avdoshenko and Makarov [74].

Note that this work is a theoretical treatment using only the geometry of a projected PES near an interesting minimum. Chemical reactions rarely exceed $1 \mathrm{~nm}$ of movement of the included atoms while mechanochemical models often involve the directional translation at a lengthscale of up to $1 \mu \mathrm{m}$ [15]. Of course, there are much more dimensions included into the pulling process than the two dimensions of interest treated here. We only pitch on the part of the pulling in the 2-dimensional plane of interest. Additionally, the mechanism through which the force, $\mathbf{f}$, is transmitted to the molecule $[12,21,51]$ is not considered here. However, finally, we have exposed a mathematical basis of a covalent mechanochemistry model with a powerful versatility using the theory of NTs.

It should also be noted that a further mathematical method produces curves which are equivalent to NTs, the Newton homotopy method $[75,76]$. It can extend the arsenal of methods to get the FDSPs curve.

\section{Conclusion}

Newton trajectories on the original PES can be used for a model of the 'reaction path following force displaced stationary points' of every developed effective PES under a pulling force. This kind of curves forms an important model for the treatment of mechanochemistry. Newton trajectories (NT) and Catastrophe theory can be used for their analysis. The theory of NTs is well prepared. Hopefully it can accomplish deeper insights into the understanding of mechanochemistry.

\section{Appendix}

We report the used formulas of the examples.

Exam 1 The surface is

$V(x, y)=4.5(1-\operatorname{Exp}[-x+1])^{2}+\left(1.75 y^{2}-0.1 y^{4}\right)$.

The minimum left below is at zero level, the last point on the $x$-axis is at level 4.34 units, where the SP on the $y$-axis is at level 7.65, and the maximum is at level 12. $D_{e}=4.5$ is the final dissociation energy, $x-1$ is the bond length displacement. 
Exam 2 The surface is

$V(x, y)=10(1-\operatorname{Exp}[-x+1])^{2}+\left(1.75 y^{2}-0.1 y^{4}\right)$.

The minimum left below is at zero level, the SP on the $x$-axis is at level 9.64 units, where the SP on the $y$-axis is at a lower level of 7.65 units.

Exam 3 The surface is an uncoupled combination of a Morse- and a quartic/sixtic potential

$V(x, y)=10(1-\operatorname{Exp}[-y+1])^{2}+\left(0.1 x^{2}+0.75 x^{4}-0.125 x^{6}\right)$.

The minimum left below is at zero level, the SP on the $x$-axis is at level 4.4 units, where the SP on the $y$-axis is at level 9.64 units, and the maximum is at 14.04 .

Exam 4 Put $h 1=1, h 2=-1.11, h 3=3, h 4=1, h 5=0, h 6=12.5$, see Ref.[8].

Form the symmetric matrices

$H 1=\left((h 1, h 2)^{T},(h 2, h 3)^{T}\right)$ and $H 2=\left((h 4, h 5)^{T},(h 5, h 6)^{T}\right)$ and put

$V(x, y)=\left[(x-1, y-1) H 1(x-1, y-1)^{T}\right]\left[(x+1, y+1) H 2(x+1, y+1)^{T}\right]$.

The minimums lie at zero level, the SP is at level 24 units, the left VRI is 145 units high, where the right VRI is at level 400 units.

Exam 5 The PES of Konda et al. [40] is

$V(x, y)=0.5 x^{2}-x^{3} / 3+0.5\left(y^{4} / 4+y^{2}(0.75-x) / 2\right)+0.2 x y^{3} / 3$.

Exam 6 It is a PES with two bound states and two SPs, corresponding to Fig. 3B of Ref.[32] $V(x, y)=(3.5+y)(1-\operatorname{Exp}[-x+1+0.2 y])^{2}+\left(-3 y^{2}+y^{4}+y^{3}\right)$.

Exam 7 The BQC surface [41] is given by
\[ V(x, y)=1 / 3\left(x^{3}-3 x y^{2}\right)-\pi(x-y)+1 / 40\left((x+7 / 4)^{4}+y^{4}\right) . \]

$$
V(x, y)=1 / 3\left(x^{3}-3 x y^{2}\right)-\pi(x-y)+1 / 40\left((x+7 / 4)^{4}+y^{4}\right) .
$$

Exam 8 The modified NFK PES [44,52] is

$$
V(x, y)=0.03\left(x^{2}+y^{2}\right)^{2}+x y-9 \operatorname{Exp}\left[-(x-3)^{2}-y^{2}\right]-9 \operatorname{Exp}\left[-(x+3)^{2}-y^{2}\right] .
$$

Acknowledgements The authors thank Peter Pain and others from the Dmug list for hints to use Mathematica.10.3 for the profile figures. There was no financial support.

\section{References}

1. M.J. Rothman, L.L. Lohr, Jr., Chem. Phys. Lett. 70, 405 (1980)

2. I.H. Williams, G.M. Maggiora, J. Mol. Struct. (Theochem) 89, 365 (1982)

3. W. Quapp, M. Hirsch, O. Imig, D. Heidrich, J. Comput. Chem. 19, 1087 (1998)

4. W. Quapp, M. Hirsch, D. Heidrich, Theor. Chem. Acc. 100(5/6), 285 (1998)

5. J.M. Anglada, E. Besalú, J.M. Bofill, R. Crehuet, J. Comput. Chem. 22, 387 (2001)

6. J.M. Bofill, J.M. Anglada, Theor. Chem. Acc. 105, 463 (2001)

7. R. Crehuet, J.M. Bofill, J.M. Anglada, Theor. Chem. Acc. 107, 130 (2002)

8. W. Quapp, J. Theoret. Comput. Chem. 2, 385 (2003)

9. W. Quapp, J. Molec. Struct. 695-696, 95 (2004)

10. J. Bofill, W. Quapp, J. Chem. Phys. 134, 074101 (2011)

11. M.K. Beyer, H. Clausen-Schaumann, Chem. Rev. 105, 2921 (2005)

12. M.M. Caruso, D.A. Davis, Q. Shen, S.A. Odom, N.R. Sottos, S.R. White, J.S. Moore, Chem. Rev. 109, 5755 (2009)

13. J.M. Lenhardt, M.T. Ong, R. Choe, C.R. Evenhuis, T.J. Martinez, S.L. Craig, Science 329, 1057 (2010)

14. Z. Huang, R. Boulatov, Chem. Soc. Rev. 40, 2359 (2011)

15. T.J. Kucharski, R. Boulatov, J. Mater. Chem. 21, 8237 (2011)

16. J. Ribas-Ariño, D. Marx, Chem. Rev. 112, 5412 (2012)

17. P. Baláž, M. Achimovičová, M. Baláž, P. Billik, Z. Cherkezova-Zheleva, et al., Chem. Soc. Rev. 42, 7571 (2013)

18. J. Wang, T.B. Kouznetsova, Z. Niuand, M.T. Ong, H.M. Klukovich, A.L. Rheingold, T.J. Martinez, S.L. Craig, Nat. Chem. 7, $323(2015)$

19. D.E. Makarov, Single Molecule Science: Physical Principles and Models (CRC Press, Taylor \& Francis Group, Boca Raton, 2015)

20. D.E. Makarov, J. Chem. Phys. 144, 030901 (2016)

21. A.L. Black, J.M. Lenhardt, S.L. Craig, J. Mater. Chem. 21, 1655 (2011)

22. S.M. Avdoshenko, D.E. Makarov, J. Phys. Chem. B (2015). DOI 10.1021/acs.jpcb.5b07613

23. Y. Tian, R. Boulatov, Chem. Commun. 49, 4187 (2013)

24. A. Bailey, N.J. Mosey, J. Chem. Phys 136, 044102 (2012)

25. S.S.M. Konda, J.M. Brantley, C.W. Bielawski, D.E. Makarov, J. Chem. Phys. 135, 164103 (2011)

26. W. Kliesch, A Mechanical String Model of Adiabatic Chemical Reactions, Lecture Notes in Chemistry, vol. 69 (Springer, Berlin, 1998)

27. Z.T. Yew, M. Schlierf, M. Rief, E. Paci, Phys. Rev. E 81, 031923 (2010)

28. V. Heinrich, A. Leung, E. Evans, J. Chem. Inf. Model. 45, 1482 (2005)

29. R.B. Best, E. Paci, G. Hummer, O.K. Dudko, J. Phys. Chem. B 112(19), 5968 (2008)

30. P.I. Zhuravlev, M. Hinczewski, S. Chakrabarti, S. Marqusee, D. Thirumalai, Proc. National Acad. Sci. pp. E715 - E724 (2016). DOI 10.1073/pnas.1515730113

31. R. Groote, R.T.M. Jakobs, R.P. Sijbesma, Polymer Chem. 4, 4864 (2013)

32. Y. Suzuki, O.K. Dudko, J. Chem. Phys. 134(6), 065102 (2011) 
33. L.B. Freund, PNAS 106, 8818 (2009)

34. F.H. Branin, IBM J. Res. Develop. 16, 504 (1972)

35. W. Quapp, J. Bofill, J. Phys. Chem. B, 120, 2644 (2016)

36. W. Quapp, J. Math Chem. 36, 365 (2004)

37. M. Hirsch, W. Quapp, J. Molec. Struct., THEOCHEM 683(1-3), 1 (2004)

38. R. Thom, Structural Stability and Morphogenesis: An Outline of a General Theory of Models (Addison-Wesley, Reading, MA, 1989)

39. D. Heidrich, W. Kliesch, W. Quapp, Properties of Chemically Interesting Potential Energy Surfaces (Springer, Berlin Heidelberg, 1991)

40. S.S.M. Konda, S.M. Avdoshenko, D.E. Makarov, J. Chem. Phys 140, 104114 (2014)

41. J.M. Bofill, W. Quapp, M. Caballero, J. Chem. Theory Comput. 8, 927 (2012)

42. D.J. Wales, T. Head-Gordon, J. Phys. Chem B 116, 8394 (2012)

43. E. Evans, A. Leung, H. Volkmar, C. Zhu, Proc. Natl. Acad. Sci. 101, 11281 (2004)

44. M. Hirsch, W. Quapp, J. Math. Chem. 36, 307 (2004)

45. S.S.M. Konda, J.M. Brantley, B.T. Varghese, K.M. Wiggins, C.W. Bielawski, D.E. Makarov, J. Am. Chem. Soc. 135, 12722 (2013)

46. V. Blanco, D.A. Leigh, V. Marcos, Chem. Soc. Rev. 44, 5341 (2015)

47. P. Valtazanos, K. Ruedenberg, Theor. Chim. Acta 69, 281 (1986)

48. W. Quapp, Theoret. Chim. Acta 75, 447 (1989)

49. J.I. Seeman, Chem. Rev. 83(2), 83 (1983)

50. V. Barsegov, D. Thirumalai, PNAS 102, 1835 (2005)

51. J.M. Lenhardt, A.L. Black, B.A. Beiermann, B.D. Steinberg, F. Rahman, T. Samborski, J. Elsakr, J.S. Moore, N.R. Sottos, S.L. Craig, J. Mater. Chem. 21, 8454 (2011)

52. E. Neria, S. Fischer, M. Karplus, J. Chem. Phys. 105, 1902 (1996)

53. D.K. Hoffmann, R.S. Nord, K. Ruedenberg, Theor. Chim. Acta 69, 265 (1986)

54. H.B. Schlegel, Theor. Chim. Acta 83, 15 (1992)

55. K. Fukui, J. Phys. Chem. 74, 4161 (1970)

56. W. Quapp, D. Heidrich, Theor. Chim. Acta 66, 245 (1984)

57. R. Crehuet, J.M. Bofill, J. Chem. Phys. 122, 234105 (2005)

58. A. Garai, Y. Zhang, O.K. Dudko, J. Chem. Phys. 140(13), 135101 (2014)

59. W. Quapp, J. Math. Chem. 54, 137 (2015)

60. M. Hirsch, W. Quapp, D. Heidrich, Phys. Chem. Chem. Phys. 1, 5291 (1999)

61. W. Quapp, V. Melnikov, Phys. Chem. Chem. Phys. 3, 2735 (2001)

62. M. Dallos, H. Lischka, E. Ventura do Monte, M. Hirsch, W. Quapp, J. Comput. Chem. 23, 276 (2002)

63. W. Quapp, D. Heidrich, J. Molec. Struct. THEOCHEM 585, 105 (2002)

64. R.M. Minyaev, I.V. Getmanskii, W. Quapp, Russ. J. Phys. Chem. 78, 1494 (2004)

65. Y. Liu, S.K. Burger, P.W. Ayers, J. Math. Chem. 49(9), 1915 (2011)

66. W. Quapp, J.M. Bofill, M. Caballero, Chem. Phys. Lett. 541, 122 (2012)

67. R.M. Minyaev, W. Quapp, B. Schmidt, I.V. Getmanski, V.V. Koval, Chem. Phys. 425, 170 (2013)

68. W. Quapp, B. Schmidt, Theor. Chem. Acc. 128, 47 (2011)

69. B. Schmidt, W. Quapp, Theor. Chem. Acc. 132, 1305 (2012)

70. W. Quapp, J. Comput. Chem 28, 1834 (2007)

71. W. Quapp, J. Bofill, A. Aguilar-Mogas, Theor. Chem. Acc. 129, 803 (2011)

72. W. Quapp, J.M. Bofill, Int. J. Quantum Chem. 115, 1635 (2015)

73. E.R. Thornton, J. Am. Chem. Soc. 89, 2915 (1967)

74. S.M. Avdoshenko, D.E. Makarov, J. Chem. Phys 142, 174106 (2015)

75. S. Ackermann, W. Kliesch, Theor. Chem. Acc. 99, 255 (1998)

76. J.M. Bofill, J. Chem. Phys. 143, 247101 (2015)

77. P. Mezey, Potential Energy Hypersurfaces (Elsevier, Amsterdam, 1987)

The graphical entry for the Table of Contents:

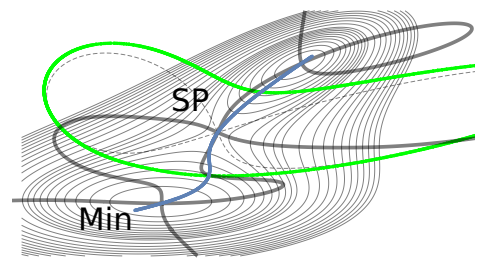

A Newton trajectory (blue) and a gradient extremal (black) cross the barrier-brakdown-line (green) at the same point. It is the point with lowest gradient with respect to other trajectories. This Newton trajectory indicates the optimal pulling direction. 